\title{
EULER-POINCARÉ FORMULATION OF HYBRID PLASMA MODELS*
}

\author{
DARRYL D. HOLM ${ }^{\dagger}$ AND CESARE TRONCI ${ }^{\ddagger}$
}

To Dave Levermore on his 60th birthday with friendship and appreciation

\begin{abstract}
Three different hybrid Vlasov-fluid systems are derived by applying reduction by symmetry to Hamilton's variational principle. In particular, the discussion focuses on the EulerPoincaré formulation of three major hybrid MHD models, which are compared in the same framework. These are the current-coupling scheme and two different variants of the pressure-coupling scheme. The Kelvin-Noether theorem is presented explicitly for each scheme, together with the Poincaré invariants for its hot particle trajectories. Extensions of Ertel's relation for the potential vorticity and for its gradient are also found in each case, as well as new expressions of cross helicity invariants.
\end{abstract}

Key words. Variational principles, Euler-Poincaré reduction, magnetohydrodynamics, MHD, Vlasov kinetic equations.

AMS subject classifications. 76M60, 76M30, 76W05, 82D10, 35Q83.

\section{Introduction}

1.1. Hybrid Vlasov-fluid models in plasma physics. Hybrid Vlasov-fluid plasma models contain elements of both continuum fluids and phase-space probability density. The latter obeys a Vlasov kinetic equation, which in turn is coupled to the momentum equation of the background magnetized fluid.

These hybrid kinetic-fluid models arise in several circumstances in modern plasma physics research, ranging from fusion research [36] to astrophysical plasmas [46]. These circumstances involve the coexistence of a cold fluid component with an ensemble of energetic particles that require a kinetic description. In meeting the challenges presented in such situations, the plasma simulation community has begun developing multiscale fluid plasma models that allow hybrid descriptions of the two types of flows. This hybrid approach successfully couples ordinary fluid models for the cold fluid component to appropriate kinetic equations that govern the phase-space distribution of the energetic particle species.

One research direction of relevance in applications is the development of hybrid schemes for magnetohydrodynamics (MHD) [36]. This development has split into two promising approaches: the current-coupling scheme [36, 1] and the pressure-coupling scheme $[36,11,8,43]$. These schemes differ in how the fluid equation is coupled to the kinetic equation for the hot particles.

Recently, the Hamiltonian formulations of a variety of hybrid Vlasov-fluid plasma models were developed that led to new theories of either current-coupled or pressurecoupled hybrid Vlasov-MHD models [44]. These Hamiltonian formulations cast considerable light on the energetics of hybrid Vlasov-fluid plasmas and the relations between their MHD approximations. In particular, the current-coupling scheme has been shown to possess a well defined Hamiltonian structure. However, the pressurecoupling schemes were found to require additional fluid transport terms in their ac-

\footnotetext{
*Received: December 3, 2010; accepted: May 24, 2011.

${ }^{\dagger}$ Department of Mathematics, Imperial College, London, United Kingdom (d.holm@imperial. ac.uk).

${ }^{\ddagger}$ Section de Mathématiques, École Polytechnique Fédérale de Lausanne, Switzerland (cesare. tronci@epfl.ch).
} 
companying kinetic equations to preserve their corresponding Hamiltonian structures, which otherwise would have been lost.

While the Hamiltonian picture of plasma models provides a powerful tool for energy-conserving properties and may also open the way to relevant stability considerations, the question has remained open whether these models allow a Lagrangian variational formulation by Hamilton's principle. Lagrangian formulations of plasma physics have been successful in several contexts, since they can be easily approached by several approximation methods such as averaging or asymptotic expansions. Well known results of this approach are contained in the Lagrangian wave theory by Dewar [10] and the celebrated guiding center motion by Littlejohn [26]. The key feature of Lagrangian variational formulations is that, in contrast to their Hamiltonian counterparts, the application of essentially any approximation scheme preserves the intrinsic geometrical properties of the resulting dynamics that emerge from its variational structure in the presence of symmetry. In particular, the derivations on the Lagrangian side provide a framework in which straight-forward application of asymptotic expansions would still preserve the fundamental circulation and Lie-Poisson properties of these theories. In contrast, asymptotic expansions of the Hamiltonian formulations, for example, require exceptional care in preserving the Jacobi identity, while applications of asymptotic expansions directly to the equations of motion typically pay no heed to these geometric properties. An illustrative example of this phenomenon for fluids was given in Camassa, Holm, and Levermore [6] in deriving the "Lake equations" and "Great Lake equations". ${ }^{1}$

The above discussion provides a natural motivation for this paper, whose aim is to present the complementary derivations of the hybrid fluid models that were introduced in [44] from the Hamiltonian side, by recovering them on the Lagrangian, or Hamilton's-principle side. As in the Hamiltonian Lie-Poisson formulation, the EulerPoincaré approach presented in this paper incorporates the geometric properties that follow from the relabeling symmetry shared by all continuum systems. In addition, the Euler-Poincaré variational framework provides a systematic framework for the derivation of other approximate models, which also inherit these geometric properties from the variational structure.

The new information we gain in this paper in each case is the natural formulation of a Kelvin-Noether circulation theorem and a corresponding Ertel theorem for the potential vorticity. While the comparisons of the Lie-Poisson Hamiltonian properties in paper [44] afford insight into the energetics of these theories, the comparisons of their complementary derivations on the Lagrangian side provide distinctions in their circulation laws, and in their Ertel relations for evolution of the potential vorticity and its gradient. Thus, the Euler-Poincaré approach affords additional insights into the diagnostics of basic MHD processes in the presence of a hot particle Vlasov component.

Maxwell-Vlasov plasmas have been treated earlier using the Euler-Poincaré variational approach based on applying symmetry reduction to Hamilton's principle [7]. The present work starts with the Low Lagrangian [27] and systematically develops a series of approximate Lagrangians for use in symmetry-reduced Hamilton's principles for re-deriving the hybrid Vlasov-MHD fluids in [44]. These approximate Lagrangians are shown to admit a variety of symmetry reductions that produce variants of Kelvin's circulation law for each theory, together with new expressions for the dynamics of their

\footnotetext{
${ }^{1}$ It was a very good moment in all our careers when Roberto Camassa, Dave Levermore, and DDH realized the efficacy of applying asymptotics to Hamilton's principle, while working on the board together one afternoon in Los Alamos.
} 
cross helicities, some of which are found to remain invariant.

The resulting Euler-Poincaré equations recover the equations in [44] and illuminate the differences in the interplay between the geometric structure and circulation mechanisms of both the current-coupled and pressure-coupled hybrid Vlasov-MHD models. The dynamics of the potential vorticity and its gradient are also explained through appropriate generalizations of Ertel's theorem to the hybrid MHD case. These generalizations arise as a direct consequence of the vorticity dynamics produced by the Euler-Poincaré equations of motion.

1.2. Plan of the paper and its main results. The main content of the paper is, as follows.

1. The remainder of this Introduction reviews the Euler-Poincaré construction of the Maxwell-Vlasov equations. Section 2 then extends this system to account for the presence of several cold fluid components (kinetic-multifluid system). The reduction process and the resulting circulation laws are presented explicitly, including the Poincaré invariant relations that are now obtained from Noether's theorem.

2. Section 3 considers the MHD limit of the kinetic-multifluid system, thereby formulating the Euler-Poincaré equations for the hybrid current-coupling MHD scheme. After presenting the Kelvin-Noether theorem, Ertel's relation for the potential vorticity is presented, thereby extending Ertel's theorem for MHD [15] to a hybrid model. Also, it is shown that the usual expression of cross helicity is conserved by this hybrid model.

3. Section 4 presents the Euler-Poincaré formulation of the first pressurecoupling hybrid MHD scheme. In this setting, the cold component drives the whole dynamics, so its velocity adds to the mean velocity of the hot particles. This property appears geometrically in the semidirect-product Lie group structure that generates the Euler-Poincaré construction. The Kelvin circulation and Ertel potential vorticity relation are derived explicitly, together with a new expression of the cross helicity invariant.

4. Section 5 focuses on the second pressure-coupling hybrid MHD scheme. In this context, the assumption of a rarefied hot component allows one to neglect the kinetic energy contribution of the corresponding mean flow. Then a decomposition becomes necessary to separate the hot particle velocity from its mean flow. This decomposition produces a nested semidirect-product Lie group structure that fits into the Euler-Poincaré construction. Explicit expressions for Kelvin circulation, Ertel's theorem and a new cross helicity invariant again result.

5. Finally Section 6 summarizes our main conclusions and discusses the outlook for future research along the present directions.

1.3. Euler-Poincaré formulation of the Maxwell-Vlasov system. The variational structure of the Maxwell-Vlasov system has been investigated in many different ways, starting from the pioneering work by Low [27]. Since then, several variational formulations of this system were presented [37, 38, 39, 47], which are mainly based on Eulerian variables. The Low Lagrangian, however, involves a mixture of Eulerian and Lagrangian variables. The first variational formulation in terms of purely Lagrangian variables appeared in [7], in which the Low Lagrangian was modified by the insertion of an extra term. This extra term ties the Lagrangian particle velocity to its corresponding Eulerian coordinate, i.e. $\dot{\mathbf{x}}\left(\mathbf{x}_{0}, \mathbf{v}_{0}\right)=\mathbf{v}\left(\mathbf{x}_{0}, \mathbf{v}_{0}\right)$. The variational 
principle was then cast into Euler-Poincaré form [21] by using a reduction process that takes advantage of the relevant symmetry properties of the Lagrangian under the Lie group of diffeomorphisms (smooth invertible maps of both physical space and phase space).

Motivated by the recent results [44] on hybrid plasma models, one may ask whether the variational methods developed in [7] would also apply to hybrid Vlasovfluid systems. This paper shows that these methods do indeed apply, and that they provide a systematic framework in which to develop a fully Lagrangian formulation of the hybrid models. The resulting theory is again an Euler-Poincaré formulation, which naturally inherits all the Lie-symmetry properties of both fluid motion and Vlasov kinetic dynamics. These symmetry properties then provide the various theorems for circulation and cross-helicity that are derived later in the paper.

The present section introduces the approach that we shall follow throughout the rest of this paper. In particular, we shall review the Euler-Poincaré variational formulation [7] of the Maxwell-Vlasov system

$$
\begin{array}{lrl}
\frac{\partial f}{\partial t}+\mathbf{v} \cdot \frac{\partial f}{\partial \mathbf{x}}+\frac{q}{m}(\mathbf{E}+\mathbf{v} \times \mathbf{B}) \cdot \frac{\partial f}{\partial \mathbf{v}}=0, & \\
\epsilon_{0} \mu_{0} \frac{\partial \mathbf{E}}{\partial t}=\nabla \times \mathbf{B}-q \mu_{0} \int \mathbf{v} f \mathrm{~d}^{3} \mathbf{v}, & \frac{\partial \mathbf{B}}{\partial t}=-\nabla \times \mathbf{E}, \\
\epsilon_{0} \nabla \cdot \mathbf{E}=q \int f \mathrm{~d}^{3} \mathbf{v}, & \nabla \cdot \mathbf{B}=0,
\end{array}
$$

where $q$ is the particle charge and $m$ its mass, while $\epsilon_{0}$ and $\mu_{0}$ are respectively the dielectric and diamagnetic constants. Moreover, in the standard notation adopted here, $f(\mathbf{x}, \mathbf{v}, t)$ is the Vlasov distribution on $\mathbb{R}^{3} \times \mathbb{R}^{3}$, while $\mathbf{E}(\mathbf{x}, t)$ and $\mathbf{B}(\mathbf{x}, t)$ are the electric field and the magnetic flux, respectively.

Euler-Poincaré approach. The Euler-Poincaré approach to the Maxwell-Vlasov system is based on an action principle of the type

$$
\delta \int_{t_{0}}^{t_{1}} L_{f_{0}}(\psi, \dot{\psi}, \Phi, \dot{\Phi}, \mathbf{A}, \dot{\mathbf{A}}) \mathrm{d} t=0
$$

where the Lagrangian $L$ is a functional

$$
L_{f_{0}}: T \operatorname{Diff}(T M) \times T \mathcal{Q} \rightarrow \mathbb{R}
$$

depending on the parameter $f_{0} \in \operatorname{Den}(T M)$, where $f_{0}$ belongs to the space of distributions on the tangent bundle $T M$ with local coordinates $\left(\mathbf{x}_{0}, \mathbf{v}_{0}\right)$. Here the notation is such that $M$ is the particle configuration space, $\psi \in \operatorname{Diff}(T M)$ is an element of the Lie group of diffeomorphisms of $T M$, and $\mathcal{Q}$ is the space of electromagnetic potentials $\left(\Phi, \mathbf{A}\right.$ ), i.e. $\mathcal{Q}=C^{\infty}(M) \times \Omega^{1}(M)$ (where $\Omega^{1}(M)$ denotes the space of differential 1 -forms on $M$ ). At this stage, the variational principle produces Euler-Lagrange equations on $\operatorname{Diff}(T M) \times \mathcal{Q}$. The explicit form of the Lagrangian reads as [7]

$$
\begin{aligned}
& L_{f_{0}}=\frac{1}{2} m \int f_{0}\left(\left|\dot{\mathbf{x}}\left(\mathbf{x}_{0}, \mathbf{v}_{0}\right)\right|^{2}+\right.\left.\left|\dot{\mathbf{x}}\left(\mathbf{x}_{0}, \mathbf{v}_{0}\right)-\mathbf{v}\left(\mathbf{x}_{0}, \mathbf{v}_{0}\right)\right|^{2}\right) \mathrm{d}^{3} \mathbf{x}_{0} \mathrm{~d}^{3} \mathbf{v}_{0} \\
&-q \int f_{0}\left(\Phi\left(\mathbf{x}\left(\mathbf{x}_{0}, \mathbf{v}_{0}\right)\right)-\dot{\mathbf{x}}\left(\mathbf{x}_{0}, \mathbf{v}_{0}\right) \cdot \mathbf{A}\left(\mathbf{x}\left(\mathbf{x}_{0}, \mathbf{v}_{0}\right)\right)\right) \mathrm{d}^{3} \mathbf{x}_{0} \mathrm{~d}^{3} \mathbf{v}_{0} \\
&+\frac{\epsilon_{0}}{2} \int\left|\nabla \Phi+\partial_{t} \mathbf{A}\right|^{2} \mathrm{~d}^{3} \boldsymbol{r}-\frac{1}{2 \mu_{0}} \int|\nabla \times \mathbf{A}|^{2} \mathrm{~d}^{3} \boldsymbol{r},
\end{aligned}
$$


where the potentials determine the electric field as $\mathbf{E}=-\nabla \Phi-\partial_{t} \mathbf{A}$ and the magnetic flux as $\mathbf{B}=\nabla \times \mathbf{A}$. Also, the map $\psi$ in the action principle above denotes $\left(\mathbf{x}\left(\mathbf{x}_{0}, \mathbf{v}_{0}\right), \mathbf{v}\left(\mathbf{x}_{0}, \mathbf{v}_{0}\right)\right):=\psi\left(\mathbf{x}_{0}, \mathbf{v}_{0}\right)$, and the term

$$
\frac{1}{2} m \int f_{0}\left|\dot{\mathbf{x}}\left(\mathbf{x}_{0}, \mathbf{v}_{0}\right)-\mathbf{v}\left(\mathbf{x}_{0}, \mathbf{v}_{0}\right)\right|^{2} \mathrm{~d}^{3} \mathbf{x}_{0} \mathrm{~d}^{3} \mathbf{v}_{0}
$$

allows $\mathbf{v}\left(\mathbf{x}_{0}, \mathbf{v}_{0}\right)$ to be varied independently and enforces $\dot{\mathbf{x}}=\mathbf{v}$. Dropping the above term returns precisely the Low Lagrangian [27].

At this point, the invariance property of the Lagrangian (1.4) is such that

$$
L_{f_{0}}(\psi, \dot{\psi}, \Phi, \dot{\Phi}, \mathbf{A}, \dot{\mathbf{A}})=L_{f_{0} \circ \psi^{-1}}\left(\dot{\psi} \circ \psi^{-1}, \Phi, \dot{\Phi}, \mathbf{A}, \dot{\mathbf{A}}\right)=: l(\mathbf{X}, \Phi, \dot{\Phi}, \mathbf{A}, \dot{\mathbf{A}}, f),
$$

where we have defined

$$
\mathbf{X}:=\dot{\psi} \circ \psi^{-1} \in \mathfrak{X}(T M), \quad f:=f_{0} \circ \psi^{-1} \in \operatorname{Den}(T M),
$$

and $\mathfrak{X}(T M)$ denotes the Lie algebra of vector fields on $T M$. Notice that the dependence on the identity element $\psi \circ \psi^{-1}$ has been omitted in the reduced Lagrangian. In this setting, the reduced Euler-Poincaré Lagrangian $l: \mathfrak{X}(T M) \times \operatorname{Den}(T M) \times T \mathcal{Q} \rightarrow \mathbb{R}$ produces the equations [7]

$$
\begin{array}{ll}
\frac{\partial}{\partial t} \frac{\delta l}{\delta \mathbf{X}}+£_{\mathbf{X}} \frac{\delta l}{\delta \mathbf{X}}=f \nabla_{(\mathbf{x}, \mathbf{v})} \frac{\delta l}{\delta f}, & \frac{\partial f}{\partial t}+£_{\mathbf{X}} f=0, \\
\frac{\partial}{\partial t} \frac{\delta l}{\delta \dot{\Phi}}-\frac{\delta l}{\delta \Phi}=0, & \frac{\partial}{\partial t} \frac{\delta l}{\delta \dot{\mathbf{A}}}-\frac{\delta l}{\delta \mathbf{A}}=0 .
\end{array}
$$

Here, the symbol $£_{\mathbf{X}}$ denotes the Lie derivative along the phase-space vector field $\mathbf{X} \in \mathfrak{X}(T M)$ whose components are given by

$$
\mathbf{X}(\mathbf{x}, \mathbf{v})=(\mathbf{u}(\mathbf{x}, \mathbf{v}), \mathbf{a}(\mathbf{x}, \mathbf{v})),
$$

in which $(\mathbf{x}, \mathbf{v}) \in T M$ are the Eulerian position-velocity coordinates and $M=\mathbb{R}^{3}$, so $\mathbf{X} \in \mathfrak{X}\left(\mathbb{R}^{6}\right)$. The symmetry-reduced version of the Lagrangian (1.4) is

$$
\begin{aligned}
l=\int f\left(\frac{1}{2} m|\mathbf{u}|^{2}+\frac{1}{2} m|\mathbf{u}-\mathbf{v}|^{2}\right. & -q \Phi+q \mathbf{u} \cdot \mathbf{A}) \mathrm{d}^{3} \mathbf{x} \mathrm{d}^{3} \mathbf{v} \\
& +\frac{\epsilon_{0}}{2} \int\left|\nabla \Phi+\partial_{t} \mathbf{A}\right|^{2} \mathrm{~d}^{3} \mathbf{x}-\frac{1}{2 \mu_{0}} \int|\nabla \times \mathbf{A}|^{2} \mathrm{~d}^{3} \mathbf{x} .
\end{aligned}
$$

The Maxwell-Vlasov equations are obtained upon applying the variations in the above Lagrangian and substituting them into the Euler-Poincaré equations (1.6)-(1.7), as shown in [7].

Outlook. The remainder of the paper applies the Euler-Poincaré approach to the case of hybrid Vlasov-fluid models that commonly arise in plasma physics research. After studying a general Vlasov-multifluid system for the interaction of several fluid plasma components with a hot particle species, the paper focuses on comparing the Euler-Poincaré structures of current-coupling and pressure-coupling hybrid MHD schemes. In the latter case, the geometry of the system provides an interesting example of how the Vlasov distribution function may be transported by the background fluid through diffeomorphisms (smooth invertible maps) acting by tangent lifts. Our considerations here are restricted to barotropic fluid flows. 
From the strictly mathematical point of view, the case of ideal adiabatic flows that transport the specific entropy may be obtained by a straightforward generalization. However, from the physical viewpoint the role of heat exchange and the effects of an additional advected quantity should lead to other interesting effects that we intend to discuss elsewhere. In particular, adiabatic flow effects may be especially interesting for hybrid fluid drift-kinetic models, which may be treated in a Lagrangian setting, perhaps by using an approach similar to that for oscillation-center theory as in [40]. For example, this problem might benefit from an exploration of adiabatic invariants that arise from averaging the Hamilton's principle. This is available for Lagrangian theories, but not for Hamiltonian theories, which instead would use Lie series methods. A proof of the equivalence of these theories would also be interesting. This approach follows ideas that go back to Dewar [10], but now have been further illuminated by the advent of the Euler-Poincare approach to reduction by symmetry for continuum descriptions on the Lagrangian side [21].

An early step in this direction was already made by Holm, Kupershmidt, and Levermore in [20], who studied Poisson maps in the Eulerian and Lagrangian descriptions of continuum mechanics. Many of the concepts from that work, particularly momentum maps from canonical phase spaces to the duals of Lie algebras, remain just as important in the present work as they were then, but here they are applied on the Lagrangian, or Hamilton's principle side for hybrid Vlasov-fluid systems.

\section{Vlasov-multifluid system}

This section presents the Euler-Poincaré formulation of a system composed of several fluid plasma species, each denoted by its label $s=1, \ldots, N$, with an energetic Vlasov component. The Hamiltonian formulation of this system has been presented in [44] and its equations of motion are expressed as

$$
\begin{aligned}
& \rho_{s} \frac{\partial \boldsymbol{U}_{s}}{\partial t}+\rho_{s}\left(\boldsymbol{U}_{s} \cdot \nabla\right) \boldsymbol{U}_{s}=a_{s} \rho_{s}\left(\mathbf{E}+\boldsymbol{U}_{s} \times \mathbf{B}\right)-\nabla \mathrm{p}_{s}, \\
& \frac{\partial \rho_{s}}{\partial t}+\nabla \cdot\left(\rho_{s} \boldsymbol{U}_{s}\right)=0, \\
& \frac{\partial f}{\partial t}+\mathbf{v} \cdot \frac{\partial f}{\partial \mathbf{x}}+a_{h}(\mathbf{E}+\mathbf{v} \times \mathbf{B}) \cdot \frac{\partial f}{\partial \mathbf{v}}=0, \\
& \mu_{0} \epsilon_{0} \frac{\partial \mathbf{E}}{\partial t}=\nabla \times \mathbf{B}-\mu_{0} \sum_{s} a_{s} \rho_{s} \boldsymbol{U}_{s}-\mu_{0} q_{h} \int \mathbf{v} f \mathrm{~d}^{3} \mathbf{v}, \\
& \frac{\partial \mathbf{B}}{\partial t}=-\nabla \times \mathbf{E}, \\
& \epsilon_{0} \nabla \cdot \mathbf{E}=\sum_{s} a_{s} \rho_{s}+q_{h} \int f \mathrm{~d}^{3} \mathbf{v}, \quad \nabla \cdot \mathbf{B}=0 .
\end{aligned}
$$

In these equations for the Vlasov-multifluid system, $a_{s}=q_{s} / m_{s}$ is the charge-to-mass ratio of the fluid species $s, \rho_{s}$ and $\boldsymbol{U}_{s}$ are its mass density and velocity, respectively, and $\mathrm{p}_{s}$ is the scalar partial pressure of species $s$. In the above system, the index $h$ denotes the hot particle component, while each fluid species $s$ is governed by its own momentum and mass-transport equations. In order to avoid proliferation of indexes, the notation $\nabla$ is relegated to denote only spatial gradients applied to quantities on physical space. Gradients on phase space coordinates are denoted by $\nabla_{(\mathbf{x}, \mathbf{v})}$, while partial differentiation of phase-space quantities will be denoted by $\partial_{\mathbf{x}}$ or $\partial_{\mathbf{v}}$.

For the case that the fluid component is absent, the Euler-Poincaré formulation of the resulting Maxwell-Vlasov system was presented in [7]. On the other hand, an 
Euler-Poincaré formulation of charged fluids was given in [21]. A combination of these two approaches yields the Euler-Poincaré formulation of the kinetic-multifluid system. Indeed, we shall show that the equations (1.6)-(1.7) can be suitably generalized to apply for the Vlasov-multifluid system. As we shall prove below, this generalization results from the following choice of Lagrangian:

$$
\begin{aligned}
& l\left(\left\{\boldsymbol{U}_{s}\right\},\left\{\rho_{s}\right\}, \mathbf{X}, f, \Phi, \dot{\Phi}, \mathbf{A}, \dot{\mathbf{A}}\right) \\
= & \frac{1}{2} \sum_{s} \int \rho_{s}\left|\boldsymbol{U}_{s}\right|^{2} \mathrm{~d}^{3} \mathbf{x}-\sum_{s} \int \rho_{s}\left(\mathcal{U}\left(\rho_{s}\right)+a_{s} \Phi-a_{s} \boldsymbol{U}_{s} \cdot \mathbf{A}\right) \mathrm{d}^{3} \mathbf{x} \\
& +m_{h} \int f\left(\frac{1}{2}|\mathbf{u}|^{2}+\frac{1}{2}|\mathbf{u}-\mathbf{v}|^{2}-a_{h} \Phi+a_{h} \mathbf{u} \cdot \mathbf{A}\right) \mathrm{d}^{3} \mathbf{x} \mathrm{d}^{3} \mathbf{v} \\
& +\frac{\epsilon_{0}}{2} \int\left|\nabla \Phi+\partial_{t} \mathbf{A}\right|^{2} \mathrm{~d}^{3} \mathbf{x}-\frac{1}{2 \mu_{0}} \int|\nabla \times \mathbf{A}|^{2} \mathrm{~d}^{3} \mathbf{x},
\end{aligned}
$$

where $\mathcal{U}\left(\rho_{s}\right)$ denotes the total internal fluid energy, related to partial pressure of species $s$ by $\mathrm{p}_{s}=\rho_{s}^{2} \mathcal{U}^{\prime}\left(\rho_{s}\right)$, while the notation $\left\{\boldsymbol{U}_{s}\right\}$ means that that one considers all species $s=1 \ldots N$ (and analogously for $\left\{\rho_{s}\right\}$ ). In the special case of a single fluid species $(s=1)$, the above Lagrangian is defined as a functional

$$
l:\left(\mathfrak{X}\left(\mathbb{R}^{3}\right) \oplus \mathfrak{X}\left(\mathbb{R}^{6}\right)\right) \times\left(C^{\infty}\left(\mathbb{R}^{3}\right)^{*} \times C^{\infty}\left(\mathbb{R}^{6}\right)^{*}\right) \times T \mathcal{Q}\left(\mathbb{R}^{3}\right) \rightarrow \mathbb{R},
$$

where $\mathfrak{X}\left(\mathbb{R}^{n}\right)$ denotes the Lie algebra of vector fields in $\mathbb{R}^{n}$, the asterisk denotes the distributional dual space, and the tangent space $T \mathcal{Q}\left(\mathbb{R}^{3}\right)$ is constructed on the space $\mathcal{Q}\left(\mathbb{R}^{3}\right)$ of electromagnetic 4-potentials $(\Phi, \mathbf{A})$. In this setting, the advected fluid quantity is the mass density $\rho(\mathbf{x}) \in \operatorname{Den}\left(\mathbb{R}^{3}\right)$, while the advected phase-space quantity is the Vlasov distribution $f(\mathbf{x}, \mathbf{v}) \in \operatorname{Den}\left(\mathbb{R}^{6}\right)$.

At this point, in order to use the above Lagrangian, equations (1.6)-(1.7) must be adapted to the present case by extending them to account for the presence of the fluid components. In following the treatment in [21, 7], one writes the Euler-Poincaré theorem in the following general form.

Theorem 2.1 (Euler-Poincaré kinetic-multifluid system). The kinetic-multifluid system (2.1)-(2.6) arises from the Euler-Poincaré variational principle

$$
\delta \int_{t_{0}}^{t_{1}} l\left(\left\{\boldsymbol{U}_{s}\right\},\left\{\rho_{s}\right\}, \mathbf{X}, f, \Phi, \dot{\Phi}, \mathbf{A}, \dot{\mathbf{A}}\right) \mathrm{d} t=0,
$$

with the Lagrangian given in (2.7) and the variations

$$
\delta \boldsymbol{U}_{s}=\partial_{t} \mathbf{W}_{s}-£_{\boldsymbol{U}_{s}} \mathbf{W}_{s}, \quad \delta \mathbf{X}=\partial_{t} \mathbf{Z}-£_{\mathbf{X}} \mathbf{Z}, \quad \delta f=-£_{\mathbf{Z}} f, \quad \delta \rho_{s}=-£_{\mathbf{W}_{s}} \rho_{s},
$$

where $\mathbf{W}_{s} \in \mathfrak{X}\left(\mathbb{R}^{3}\right), \mathbf{Z} \in \mathfrak{X}\left(\mathbb{R}^{6}\right), \delta \Phi$, and $\delta \mathbf{A}$ all vanish at the endpoints. This variational principle is equivalent to the Euler-Poincaré equations

$$
\begin{aligned}
& \frac{\partial}{\partial t} \frac{\delta l}{\delta \boldsymbol{U}_{s}}+£_{\boldsymbol{U}_{s}} \frac{\delta l}{\delta \boldsymbol{U}_{s}}=\rho_{s} \nabla \frac{\delta l}{\delta \rho_{s}}, \\
& \frac{\partial}{\partial t} \frac{\delta l}{\delta \mathbf{X}}+£_{\mathbf{X}} \frac{\delta l}{\delta \mathbf{X}}=f \nabla_{(\mathbf{x}, \mathbf{v})} \frac{\delta l}{\delta f}, \\
& \frac{\partial \rho_{s}}{\partial t}+£_{\boldsymbol{U}_{s}} \rho_{s}=0, \quad \frac{\partial f}{\partial t}+£_{\mathbf{X}} f=0, \\
& \frac{\partial}{\partial t} \frac{\delta l}{\delta \dot{\Phi}}-\frac{\delta l}{\delta \Phi}=0, \quad \frac{\partial}{\partial t} \frac{\delta l}{\delta \dot{\mathbf{A}}}-\frac{\delta l}{\delta \mathbf{A}}=0 .
\end{aligned}
$$


Proof. The equivalence of the variational principle (2.8) and the Euler-Poincaré equations (2.9)-(2.12) follows easily upon repeating the same steps as in [21, 7]. In order to derive the equations (2.1)-(2.6), one simply computes the functional derivatives of the Lagrangian (2.7). In particular, upon writing $\mathbf{X}=(\mathbf{u}, \mathbf{a})$, for the Vlasov kinetic part one has

$$
\frac{\delta l}{\delta \mathbf{u}}=m_{h} f\left(2 \mathbf{u}-\mathbf{v}+a_{h} \mathbf{A}\right), \quad \frac{\delta l}{\delta \mathbf{a}}=0, \quad \frac{\delta l}{\delta f}=\frac{m_{h}}{2}|\mathbf{u}|^{2}+\frac{m_{h}}{2}|\mathbf{u}-\mathbf{v}|^{2}+q_{h} \mathbf{u} \cdot \mathbf{A}-q_{h} \Phi .
$$

Then, dividing equation (2.10) by $f$ yields

$$
\frac{\partial}{\partial t}\left(\frac{1}{f} \frac{\delta l}{\delta \mathbf{X}}\right)+£_{\mathbf{X}}\left(\frac{1}{f} \frac{\delta l}{\delta \mathbf{X}}\right)=\nabla_{(\mathbf{x}, \mathbf{v})} \frac{\delta l}{\delta f} .
$$

Next, projecting onto the second component yields

$$
0=\partial_{\mathbf{v}} \mathbf{u} \cdot \frac{1}{f} \frac{\delta l}{\delta \mathbf{u}}-\partial_{\mathbf{v}} \frac{\delta l}{\delta f}=\mathbf{u}(\mathbf{x}, \mathbf{v})-\mathbf{v},
$$

so that

$$
\mathbf{X}(\mathbf{x}, \mathbf{v})=(\mathbf{v}, \mathbf{a}(\mathbf{x}, \mathbf{v})), \quad \frac{\delta l}{\delta \mathbf{u}}=f\left(m_{h} \mathbf{v}+q_{h} \mathbf{A}\right), \quad \frac{\delta l}{\delta f}=\frac{m_{h}}{2}|\mathbf{v}|^{2}+q_{h} \mathbf{v} \cdot \mathbf{A}-q_{h} \Phi .
$$

Upon denoting the particle momentum as $\boldsymbol{p}(\mathbf{x}, \mathbf{v}):=m_{h} \mathbf{v}+q_{h} \mathbf{A}(\mathbf{x})$ and projecting equation (2.13) onto its first component, we obtain

$$
\partial_{t} \boldsymbol{p}+\left(\mathbf{v} \cdot \partial_{\mathbf{x}}+\mathbf{a} \cdot \partial_{\mathbf{v}}\right) \boldsymbol{p}+\partial_{\mathbf{x}} \mathbf{v} \cdot \boldsymbol{p}=\partial_{\mathbf{x}}\left(\frac{m_{h}}{2}|\mathbf{v}|^{2}+q_{h} \mathbf{v} \cdot \mathbf{A}-q_{h} \Phi\right),
$$

where $(\mathbf{x}, \mathbf{v})$ are independent coordinates. Standard vector identities then produce the Lorentz force,

$$
\mathbf{a}(\mathbf{x}, \mathbf{v})=-a_{h}\left(\nabla \Phi+\frac{\partial \mathbf{A}}{\partial t}\right)+a_{h} \mathbf{v} \times(\nabla \times \mathbf{A}) .
$$

Therefore, the second equation of (2.11) gives the Vlasov kinetic equation in the form

$$
\frac{\partial f}{\partial t}+\mathbf{v} \cdot \frac{\partial f}{\partial \mathbf{x}}-a_{h}\left[\left(\nabla \Phi+\frac{\partial \mathbf{A}}{\partial t}\right)-\mathbf{v} \times(\nabla \times \mathbf{A})\right] \cdot \frac{\partial f}{\partial \mathbf{v}}=0 .
$$

The fluid equations follow easily by inserting the appropriate variational derivatives into the Euler-Poincaré equation (2.9) and the first equation of (2.11). For example, one computes

$$
\frac{\delta l}{\delta \boldsymbol{U}_{s}}=\rho_{s} \boldsymbol{U}_{s}+a_{s} \rho_{s} \mathbf{A}, \quad \frac{\delta l}{\delta \rho_{s}}=-\frac{\mathrm{d}\left(\rho_{s} \mathcal{U}\right)}{\mathrm{d} \rho_{s}}-a_{s} \Phi+a_{s} \boldsymbol{U} \cdot \mathbf{A} .
$$

Next, upon dividing equation (2.9) by $\rho$, one obtains

$$
\frac{\partial \boldsymbol{U}_{s}}{\partial t}+£_{\boldsymbol{U}_{s}} \boldsymbol{U}_{s}+a_{s}\left(\frac{\partial \mathbf{A}}{\partial t}+£_{\boldsymbol{U}_{s}} \mathbf{A}\right)=-\nabla \mathrm{p}_{s}-a_{s} \nabla \Phi+a_{s} \nabla\left(\boldsymbol{U}_{s} \cdot \mathbf{A}\right),
$$

where $\mathrm{p}_{s}=\rho_{s}^{2} \mathcal{U}^{\prime}\left(\rho_{s}\right)$ is the scalar partial pressure. Finally, equation (2.1) arises from the explicit form of the Lie derivative operation by using standard vector identities. Analogous arguments also hold for the equations of the electromagnetic potentials. $\square$ 
REMARK 2.2 (Euler-Poincaré reduction). The above theorem follows by an EulerPoincaré reduction process applied to the following unreduced Lagrangian:

$$
\begin{aligned}
& \quad L_{f_{0},\left\{\rho_{0 s}\right\}}\left(\left\{\eta_{s}\right\},\left\{\dot{\eta}_{s}\right\}, \psi, \dot{\psi}, \Phi, \dot{\Phi}, \mathbf{A}, \dot{\mathbf{A}}\right) \\
& =\frac{1}{2} \sum_{s} \int \rho_{0 s}\left(\boldsymbol{a}_{0}\right)\left|\dot{\eta}_{s}\left(\boldsymbol{a}_{0}\right)\right|^{2} \mathrm{~d}^{3} \boldsymbol{a}_{0} \\
& \quad-\sum_{s} \int \rho_{0 s}\left(\boldsymbol{a}_{0}\right)\left(\mathcal{U}\left(\rho_{0 s}\right)+a_{s} \Phi\left(\eta_{s}\left(\boldsymbol{a}_{0}\right)\right)-a_{s} \dot{\eta}_{s}\left(\boldsymbol{a}_{0}\right) \cdot \mathbf{A}\left(\eta_{s}\left(\boldsymbol{a}_{0}\right)\right)\right) \mathrm{d}^{3} \boldsymbol{a}_{0} \\
& \quad+\frac{1}{2} m_{h} \int f_{0}\left(\mathbf{x}_{0}, \mathbf{v}_{0}\right)\left(\left|\dot{\boldsymbol{q}}\left(\mathbf{x}_{0}, \mathbf{v}_{0}\right)\right|^{2}+\left|\dot{\boldsymbol{q}}\left(\mathbf{x}_{0}, \mathbf{v}_{0}\right)-\boldsymbol{v}\left(\mathbf{x}_{0}, \mathbf{v}_{0}\right)\right|^{2}\right) \mathrm{d}^{3} \mathbf{x}_{0} \mathrm{~d}^{3} \mathbf{v}_{0} \\
& \quad-q_{h} \int f_{0}\left(\mathbf{x}_{0}, \mathbf{v}_{0}\right)\left(\Phi\left(\boldsymbol{q}\left(\mathbf{x}_{0}, \mathbf{v}_{0}\right)\right)+\dot{\boldsymbol{q}}\left(\mathbf{x}_{0}, \mathbf{v}_{0}\right) \cdot \mathbf{A}\left(\boldsymbol{q}\left(\mathbf{x}_{0}, \mathbf{v}_{0}\right)\right)\right) \mathrm{d}^{3} \mathbf{x}_{0} \mathrm{~d}^{3} \mathbf{v}_{0} \\
& \quad+\frac{\epsilon_{0}}{2} \int\left|\nabla \Phi+\partial_{t} \mathbf{A}\right|^{2} \mathrm{~d}^{3} \mathbf{r}-\frac{1}{2 \mu_{0}} \int|\nabla \times \mathbf{A}|^{2} \mathrm{~d}^{3} \mathbf{r},
\end{aligned}
$$

with the notation $\left(\boldsymbol{q}\left(\mathbf{x}_{0}, \mathbf{v}_{0}\right), \boldsymbol{v}\left(\mathbf{x}_{0}, \mathbf{v}_{0}\right)\right)=\psi\left(\mathbf{x}_{0}, \mathbf{v}_{0}\right)$ for a group action $\psi: T \mathbb{R}^{3} \rightarrow T \mathbb{R}^{3}$. Indeed, the invariance property

$$
L_{f_{0},\left\{\rho_{0 s}\right\}}\left(\left\{\eta_{s}\right\},\left\{\dot{\eta}_{s}\right\}, \psi, \dot{\psi}, \Phi, \dot{\Phi}, \mathbf{A}, \dot{\mathbf{A}}\right)=L_{f_{0} \circ \psi^{-1},\left\{\rho_{0 s} \circ \eta_{s}^{-1}\right\}}\left(\left\{\dot{\eta}_{s} \circ \eta_{s}^{-1}\right\}, \dot{\psi} \circ \psi^{-1}, \Phi, \dot{\Phi}, \mathbf{A}, \dot{\mathbf{A}}\right)
$$

yields the Euler-Poincaré Lagrangian

$$
l\left(\left\{\boldsymbol{U}_{s}\right\},\left\{\rho_{s}\right\}, \mathbf{X}, f, \Phi, \dot{\Phi}, \mathbf{A}, \dot{\mathbf{A}}\right):=L_{f_{0} \circ \psi^{-1},\left\{\rho_{0 s} \circ \eta_{s}^{-1}\right\}}\left(\left\{\dot{\eta}_{s} \circ \eta_{s}^{-1}\right\}, \dot{\psi} \circ \psi^{-1}, \Phi, \dot{\Phi}, \mathbf{A}, \dot{\mathbf{A}}\right),
$$

with the notation

$$
\boldsymbol{U}_{s}=\dot{\eta}_{s} \circ \eta_{s}^{-1}, \quad \mathbf{X}=\dot{\psi} \circ \psi^{-1}, \quad \rho_{s}=\rho_{0 s} \circ \eta_{s}^{-1}, \quad \text { and } \quad f=f_{0} \circ \psi^{-1} .
$$

This argument follows easily from the treatment in [21, 7]. Notice that for the case of a single species $s=1$, the unreduced Lagrangian is of the form

$$
L_{f_{0}, \rho_{0}}: T \operatorname{Diff}\left(\mathbb{R}^{3}\right) \times T \operatorname{Diff}\left(T \mathbb{R}^{3}\right) \times T \mathcal{Q} \rightarrow \mathbb{R},
$$

which emphasizes the Lie group structure that underlies the Vlasov-multifluid system (2.1)-(2.6)

REMARK 2.3 (Kelvin-Noether theorem for the Vlasov multifluid system). It is easy to verify that equations (2.9)-(2.10) produce the following circulation conservation laws:

$$
\frac{\mathrm{d}}{\mathrm{d} t} \oint_{\gamma_{t}\left(\boldsymbol{U}_{s}\right)}\left(\boldsymbol{U}_{s}(\mathbf{x}, t)+a_{s} \mathbf{A}(\mathbf{x}, t)\right) \cdot \mathrm{d} \mathbf{x}=0, \quad \frac{\mathrm{d}}{\mathrm{d} t} \oint_{\zeta_{t}(\mathbf{X})}\left(\mathbf{v}+a_{h} \mathbf{A}(\mathbf{x}, t)\right) \cdot \mathrm{d} \mathbf{x}=0 .
$$

In the first relation the curve $\gamma_{t}$ moves with the fluid flow, while in the second relation the curve $\zeta_{t}$ moves with the phase-space vector field $\mathbf{X}(\mathbf{x}, \mathbf{v})=(\mathbf{v}, \mathbf{a}(\mathbf{x}, \mathbf{v}))$. More explicitly, one can write $\gamma_{t}=\eta_{s}(t) \circ \gamma_{0}$ for a fixed loop $\gamma_{0}$, and analogously $\zeta_{t}=\psi(t) \circ \zeta_{0}$. This difference emphasizes the role of the Poincaré invariant associated with the hot particle motion; see [7].

The next sections will consider the Euler-Poincaré formulation of hybrid VlasovMHD models. In particular, the discussion will focus on two main types of hybrid systems: the current-coupling and pressure-coupling schemes. 
Remark 2.4 (The Legendre transform). Notice that the Euler-Poincaré Lagrangian (2.7) is degenerate since $\delta l / \delta \mathbf{a}=0$. This degeneracy is related to a redundancy in the Euler-Poincaré construction, which carries all the information about particle paths that are already encoded in the Vlasov equation. As explained in [7], this degeneracy presents some problems when one wants to perform a Legendre transform to obtain the corresponding Hamiltonian description. However, these problems may be overcome by a standard use of Dirac constraints. Similar arguments to those in [7] also hold for the hybrid models treated in this paper.

\section{Current-coupling hybrid MHD scheme}

3.1. Formulation of the model. In common physical situations, one is interested in single-fluid models. In the context of hybrid schemes, it is customary to specialize the system (2.1)-(2.6) to the two-fluid case and to neglect the inertia of one of the fluid species (electrons). This last approximation is equivalent to taking the limit $m_{2} \rightarrow 0$ for the second species in the total fluid momentum equation. Under this assumption, the sum of the equation (2.1) for $s=1,2$ produces

$$
\rho_{1} \frac{\partial \boldsymbol{U}_{1}}{\partial t}+\rho_{1}\left(\boldsymbol{U}_{1} \cdot \nabla\right) \boldsymbol{U}_{1}=\left(a_{1} \rho_{1}+a_{2} \rho_{2}\right) \mathbf{E}+\left(a_{1} \rho_{1} \boldsymbol{U}_{1}+a_{2} \rho_{2} \boldsymbol{U}_{2}\right) \times \mathbf{B}-\nabla \mathrm{p}_{1} .
$$

Also, upon assuming neutrality by letting $\epsilon_{0} \rightarrow 0$, the electromagnetic fields satisfy the equations

$$
\begin{aligned}
& \sum_{s} a_{s} \rho_{s} \boldsymbol{U}_{s}=\frac{1}{\mu_{0}} \nabla \times \mathbf{B}-a_{h} \int \mathbf{v} f \mathrm{~d}^{3} \mathbf{v}, \\
& \frac{\partial \mathbf{B}}{\partial t}=-\nabla \times \mathbf{E}, \\
& \sum_{s} a_{s} \rho_{s}=-q_{h} \int f \mathrm{~d}^{3} \mathbf{v}, \quad \nabla \cdot \mathbf{B}=0 .
\end{aligned}
$$

Then, equation (3.1) becomes

$$
\rho \frac{\partial \boldsymbol{U}}{\partial t}+\rho(\boldsymbol{U} \cdot \nabla) \boldsymbol{U}=-\left(q_{h} \int f \mathrm{~d}^{3} \mathbf{v}\right) \mathbf{E}+\left(\frac{1}{\mu_{0}} \nabla \times \mathbf{B}-a_{h} \int \mathbf{v} f \mathrm{~d}^{3} \mathbf{v}\right) \times \mathbf{B}-\nabla \mathbf{p},
$$

where we have dropped labels for convenience. Finally, inserting Ohm's ideal law $\mathbf{E}+\boldsymbol{U} \times \mathbf{B}=0$, the kinetic two-fluid system becomes

$$
\begin{aligned}
& \rho \frac{\partial \boldsymbol{U}}{\partial t}+\rho(\boldsymbol{U} \cdot \nabla) \boldsymbol{U}=\left(q_{h} \boldsymbol{U} \int f \mathrm{~d}^{3} \mathbf{v}-q_{h} \int \mathbf{v} f \mathrm{~d}^{3} \mathbf{v}+\frac{1}{\mu_{0}} \nabla \times \mathbf{B}\right) \times \mathbf{B}-\nabla \mathbf{p} \\
& \frac{\partial \rho}{\partial t}+\nabla \cdot(\rho \boldsymbol{U})=0 \\
& \frac{\partial f}{\partial t}+\mathbf{v} \cdot \frac{\partial f}{\partial \mathbf{x}}+a_{h}(\mathbf{v}-\boldsymbol{U}) \times \mathbf{B} \cdot \frac{\partial f}{\partial \mathbf{v}}=0 \\
& \frac{\partial \mathbf{B}}{\partial t}=\nabla \times(\boldsymbol{U} \times \mathbf{B}) .
\end{aligned}
$$

This is the same as the current-coupling hybrid scheme presented in [11,36, 1], except that the particle dynamics are governed by the Vlasov equation rather than its gyrokinetic counterpart. Notice that the above system does not make any assumption about the form of the Vlasov distribution for the energetic particles. Therefore, this system should in principle apply to a variety of other possible physical situations as well. 
3.2. Euler-Poincaré reduction by symmetry. We now turn our attention to the Euler-Poincaré formulation of these equations. That is, we ask whether the above current-coupling system possesses an Euler-Poincaré variational principle. A positive answer is provided by the reduced Lagrangian

$$
\begin{aligned}
l(\boldsymbol{U}, \rho, \mathbf{X}, f, \mathbf{A})=\frac{1}{2} \int \rho|\boldsymbol{U}|^{2} \mathrm{~d}^{3} \mathbf{x}-\int \rho \mathcal{U}(\rho) \mathrm{d}^{3} \mathbf{x}-\frac{1}{2 \mu_{0}} \int|\nabla \times \mathbf{A}|^{2} \mathrm{~d}^{3} \mathbf{x} \\
+\int f\left(\frac{m_{h}}{2}|\mathbf{u}|^{2}+\frac{m_{h}}{2}|\mathbf{u}-\mathbf{v}|^{2}+q_{h}(\mathbf{u}-\boldsymbol{U}) \cdot \mathbf{A}\right) \mathrm{d}^{3} \mathbf{x} \mathrm{d}^{3} \mathbf{v}
\end{aligned}
$$

of the type

$$
l:\left(\mathfrak{X}\left(\mathbb{R}^{3}\right) \oplus \mathfrak{X}\left(\mathbb{R}^{6}\right)\right) \times C^{\infty}\left(\mathbb{R}^{3}\right)^{*} \times \Omega^{1}\left(\mathbb{R}^{3}\right) \times C^{\infty}\left(\mathbb{R}^{6}\right)^{*} \rightarrow \mathbb{R},
$$

together with the following Euler-Poincaré theorem.

THEOREM 3.1. The hybrid current-coupling MHD scheme (3.6)-(3.9) arises from the Euler-Poincaré variational principle

$$
\delta \int_{t_{0}}^{t_{1}} l(\boldsymbol{U}, \rho, \mathbf{X}, f, \mathbf{A}) \mathrm{d} t=0,
$$

with the Lagrangian in (3.10) and variations given by

$$
\delta \boldsymbol{U}=\partial_{t} \mathbf{W}-£_{\boldsymbol{U}} \mathbf{W}, \quad \delta \mathbf{X}=\partial_{t} \mathbf{Z}-£_{\mathbf{X}} \mathbf{Z}, \quad \delta f=-£_{\mathbf{Z}} f, \quad \delta \rho=-£_{\boldsymbol{W}} \rho, \quad \delta \mathbf{A}=-£_{\boldsymbol{W}} \mathbf{A},
$$

where $\mathbf{W}$ and $\mathbf{Z}$ vanish at the endpoints. This variational principle is equivalent to the Euler-Poincaré equations

$$
\begin{aligned}
& \frac{\partial}{\partial t} \frac{\delta l}{\delta \boldsymbol{U}}+£_{\boldsymbol{U}} \frac{\delta l}{\delta \boldsymbol{U}}=\rho \nabla \frac{\delta l}{\delta \rho}-\frac{\delta l}{\delta \mathbf{A}} \times(\nabla \times \mathbf{A})+\left(\nabla \cdot \frac{\delta l}{\delta \mathbf{A}}\right) \mathbf{A}, \\
& \frac{\partial \rho}{\partial t}+£_{\boldsymbol{U}} \rho=0, \quad \frac{\partial \mathbf{A}}{\partial t}+£_{\boldsymbol{U}} \mathbf{A}=0, \\
& \frac{\partial}{\partial t} \frac{\delta l}{\delta \mathbf{X}}+£_{\mathbf{X}} \frac{\delta l}{\delta \mathbf{X}}=f \nabla_{(\mathbf{x}, \mathbf{v})} \frac{\delta l}{\delta f} \\
& \frac{\partial f}{\partial t}+£_{\mathbf{X}} f=0,
\end{aligned}
$$

which hold for an arbitrary hybrid Lagrangian.

Proof. The derivation of the Euler-Poincaré equations (3.11)-(3.14) from the Euler-Poincaré variational principle can be easily obtained by direct verification $[21,7]$. In order to derive the current-coupling MHD scheme (3.6)-(3.9), one simply computes the functional derivatives and inserts them into the Euler-Poincaré equations (3.11)-(3.14). In particular, for the Vlasov kinetic part one has

$$
\frac{\delta l}{\delta \mathbf{u}}=m_{h} f\left(2 \mathbf{u}-\mathbf{v}+a_{h} \mathbf{A}\right), \frac{\delta l}{\delta \mathbf{a}}=0, \quad \frac{\delta l}{\delta f}=\frac{m_{h}}{2}|\mathbf{u}|^{2}+\frac{m_{h}}{2}|\mathbf{u}-\mathbf{v}|^{2}+q_{h}(\mathbf{u}-\boldsymbol{U}) \cdot \mathbf{A} .
$$

Then, by projecting equation (2.10) onto the second component (recall that $\mathbf{X}=$ $(\mathbf{u}, \mathbf{a}))$, we get

$$
0=\partial_{\mathbf{v}} \mathbf{u} \cdot \frac{1}{f} \frac{\delta l}{\delta \mathbf{u}}-\partial_{\mathbf{v}} \frac{\delta l}{\delta f}=\mathbf{u}(\mathbf{x}, \mathbf{v})-\mathbf{v}
$$


so that

$$
\mathbf{X}(\mathbf{x}, \mathbf{v})=(\mathbf{v}, \mathbf{a}(\mathbf{x}, \mathbf{v})), \quad \frac{\delta l}{\delta \mathbf{u}}=m_{h} f\left(\mathbf{v}+a_{h} \mathbf{A}\right), \quad \frac{\delta l}{\delta f}=\frac{m_{h}}{2}|\mathbf{v}|^{2}+q_{h}(\mathbf{v}-\boldsymbol{U}) \cdot \mathbf{A} .
$$

Upon denoting $\boldsymbol{p}(\mathbf{x}, \mathbf{v})=m_{h} \mathbf{v}+q_{h} \mathbf{A}(\mathbf{x})$ and dividing equation (2.10) by $f$, one finds

$$
\frac{\partial}{\partial t}\left(\frac{1}{f} \frac{\delta l}{\delta \mathbf{X}}\right)+£_{\mathbf{X}}\left(\frac{1}{f} \frac{\delta l}{\delta \mathbf{X}}\right)=\nabla_{(\mathbf{x}, \mathbf{v})} \frac{\delta l}{\delta f}
$$

which when projected onto the first component yields

$$
\partial_{t} \boldsymbol{p}+\left(\mathbf{v} \cdot \partial_{\mathbf{x}}+\mathbf{a} \cdot \partial_{\mathbf{v}}\right) \boldsymbol{p}+\partial_{\mathbf{x}} \mathbf{v} \cdot \boldsymbol{p}=\partial_{\mathbf{x}}\left(\frac{m_{h}}{2}|\mathbf{v}|^{2}+q_{h}(\mathbf{v}-\boldsymbol{U}) \cdot \mathbf{A}\right) .
$$

Upon recalling that $(\mathbf{x}, \mathbf{v})$ are independent coordinates, and using standard vector identities, we can write

$$
\begin{aligned}
\mathbf{a}(\mathbf{x}, \mathbf{v}) & =-a_{h}\left(\nabla(\boldsymbol{U} \cdot \mathbf{A})+\frac{\partial \mathbf{A}}{\partial t}\right)+a_{h} \mathbf{v} \times(\nabla \times \mathbf{A}), \\
& =a_{h}(\mathbf{v}-\boldsymbol{U}) \times(\nabla \times \mathbf{A})
\end{aligned}
$$

where the bottom line is justified by the second equation in (3.12). Therefore, equation (3.14) returns the Vlasov kinetic equation (3.8) in the form

$$
\frac{\partial f}{\partial t}+\mathbf{v} \cdot \frac{\partial f}{\partial \mathbf{x}}+a_{h}[(\mathbf{v}-\boldsymbol{U}) \times(\nabla \times \mathbf{A})] \cdot \frac{\partial f}{\partial \mathbf{v}}=0
$$

with a modified Lorentz force.

We now focus on the fluid part. It suffices to compute

$$
\frac{\delta l}{\delta \boldsymbol{U}}=\rho \boldsymbol{U}-q_{h} n \mathbf{A}, \quad \frac{\delta l}{\delta \mathbf{A}}=-\nabla \times \nabla \times \mathbf{A}+q_{h}(\mathbf{K}-n \boldsymbol{U}), \quad \frac{\delta l}{\delta \rho}=\frac{1}{2}|\boldsymbol{U}|^{2}+\rho \mathcal{U}^{\prime}(\rho)+\mathcal{U}(\rho),
$$

where we have introduced the additional notation

$$
n=\int f \mathrm{~d}^{3} \mathbf{v}, \quad \mathbf{K}=\int \mathbf{v} f \mathrm{~d}^{3} \mathbf{v} .
$$

At this point, it suffices to insert the above functional derivatives into equation (3.11), so that

$$
\begin{aligned}
\left(\frac{\partial}{\partial t}+£_{\boldsymbol{U}}\right)\left(\rho \boldsymbol{U}-q_{h} n \mathbf{A}\right)=\rho & \nabla \\
& \left(\frac{1}{2}|\boldsymbol{U}|^{2}+\rho \mathcal{U}^{\prime}(\rho)+\mathcal{U}(\rho)\right) \\
& -q_{h}(\mathbf{K}-n \boldsymbol{U}) \times \nabla \times \mathbf{A}+q_{h} \mathbf{A} \nabla \cdot(\mathbf{K}-n \boldsymbol{U}) \\
& +(\nabla \times \nabla \times \mathbf{A}) \times(\nabla \times \mathbf{A}) .
\end{aligned}
$$

We observe that the zero-th moment of the Vlasov equation (3.8) satisfies $\partial_{t} n+\nabla \cdot \mathbf{K}=0$. Then, making use of the second equation in (3.12) yields

$$
\left(\partial_{t}+£_{\boldsymbol{U}}\right)(n \mathbf{A})=-\mathbf{A} \nabla \cdot(\mathbf{K}-n \boldsymbol{U})
$$

while expanding the Lie derivatives in (3.15) returns the velocity equation

$$
\rho \frac{\partial \boldsymbol{U}}{\partial t}+\rho(\boldsymbol{U} \cdot \nabla) \boldsymbol{U}=\left(q_{h} n \boldsymbol{U}-q_{h} \mathbf{K}+\frac{1}{\mu_{0}} \nabla \times \mathbf{B}\right) \times \mathbf{B}-\nabla \mathbf{p},
$$


in which we have substituted $\mathbf{B}=\nabla \times \mathbf{A}$ and $\mathbf{p}=\rho^{2} \mathcal{U}^{\prime}(\rho)$.

REMARK 3.2 (Euler-Poincaré reduction). Upon following the treatment in [21, 7], one finds that the unreduced Euler-Poincaré Lagrangian of the current-coupling scheme is a functional of the type

$$
L_{\rho_{0}, \mathbf{A}_{0}, f_{0}}: T \operatorname{Diff}\left(\mathbb{R}^{3}\right) \times T \operatorname{Diff}\left(\mathbb{R}^{6}\right) \rightarrow \mathbb{R},
$$

where $\times$ denotes direct product. Consequently,

$$
L_{\rho_{0}, \mathbf{A}_{0}, f_{0}}=L_{\rho_{0}, \mathbf{A}_{0}, f_{0}}(\eta, \dot{\eta}, \psi, \dot{\psi}) .
$$

The reduced Euler-Poincaré Lagrangian (3.10) is obtained by the reduction process

$$
l(\mathbf{u}, \mathbf{X}, \rho, \mathbf{A}, f)=L_{\rho_{0} \circ \eta^{-1}, \mathbf{A}_{0} \circ \eta^{-1}, f_{0} \circ \psi^{-1}}\left(\dot{\eta} \circ \eta^{-1}, \dot{\psi} \circ \psi^{-1}\right) .
$$

Here the advected quantities $\rho, \mathbf{A}, f$ are acted on by the corresponding diffeomorphism groups, taking into account their intrinsic tensorial nature - that is $(\rho, \mathbf{A}, f) \in$ $\operatorname{Den}\left(\mathbb{R}^{3}\right) \times \Omega^{1}\left(\mathbb{R}^{3}\right) \times \operatorname{Den}\left(\mathbb{R}^{6}\right)$, where $\Omega^{1}\left(\mathbb{R}^{3}\right)$ denotes the space of differential oneforms on $\mathbb{R}^{3}$.

3.3. Discussion of the Kelvin circulation law. Relation (3.15) amounts to the following Kelvin circulation law:

$$
\begin{aligned}
\frac{\mathrm{d}}{\mathrm{d} t} \oint_{\gamma_{t}(\boldsymbol{U})}\left(\boldsymbol{U}-q_{h} \frac{n}{\rho} \mathbf{A}\right) \cdot \mathrm{d} \mathbf{x}=q_{h} \oint_{\gamma_{t}(\boldsymbol{U})} \frac{1}{\rho}((\nabla \cdot(\mathbf{K}-n \boldsymbol{U})) \mathbf{A}-(\mathbf{K}-n \boldsymbol{U}) \times \mathbf{B}) \cdot \mathrm{d} \mathbf{x} \\
+\oint_{\gamma_{t}(\boldsymbol{U})} \frac{1}{\rho}(\nabla \times \mathbf{B}) \times \mathbf{B} \cdot \mathrm{d} \mathbf{x}, \quad
\end{aligned}
$$

which agrees with the corresponding result found in [44]. Notice that the creation of circulation on the right hand side is generated by the terms involving $\delta l / \delta \mathbf{A}$ in the Euler-Poincaré equation (3.11). As explained in [21], these terms comprise a momentum map generated by the action of the diffeomorphisms on the cotangent bundle $T^{*} \Omega^{1}(\mathbb{R})$. The presence of these terms is related to the fact that the nonzero magnetic potential A (together with the mass density $\rho$ ) breaks the relabeling symmetry of the unreduced Lagrangian, so that

$$
L_{\rho_{0}, \mathbf{A}_{0}, f_{0}}(\eta, \dot{\eta}, \psi, \dot{\psi}) \neq L_{\rho_{0} \circ \eta^{-1}, \mathbf{A}_{0}, f_{0} \circ \psi^{-1}}\left(\dot{\eta} \circ \eta^{-1}, \psi, \dot{\psi}\right) .
$$

On the other hand, the Kelvin circulation theorem for the hot particles reads simply

$$
\frac{\mathrm{d}}{\mathrm{d} t} \oint_{\zeta_{t}(\mathbf{X})} \boldsymbol{p} \cdot \mathrm{d} \mathbf{x}=0
$$

which recovers the well known preservation of the Poincaré invariant for the hot particle motion.

Ertel's theorem. The above Kelvin circulation law identifies the expression of the force

$$
\mathbf{\Psi}=(\nabla \cdot(\mathbf{K}-n \boldsymbol{U})) \mathbf{A}-(\mathbf{K}-n \boldsymbol{U}) \times \mathbf{B}+\mu_{0}^{-1}(\nabla \times \mathbf{B}) \times \mathbf{B}
$$

acting on the fluid with momentum $\rho \boldsymbol{U}-q_{h} n \mathbf{A}$. The above quantity can be used to generalize Ertel's theorem for MHD (see [15] and references therein) to the hybrid 
current-coupling scheme. For simplicity, consider the incompressible case, so that $\rho \equiv 1$ enforces $\nabla \cdot \boldsymbol{U}=0$. Next, project the quantity $\rho^{-1} n \mathbf{A}$ onto its divergence-free part by defining $\left[\rho^{-1} n \mathbf{A}\right]=\rho^{-1} n \mathbf{A}+\nabla \varphi$, for a scalar function $\varphi$ such that $\nabla \cdot\left[\rho^{-1} n \mathbf{A}\right]=0$. Notice that we keep the density $\rho$ in these relations to provide correct dimensions, while $\rho=1$ for incompressible flows. Then, upon denoting $D_{t}=\partial_{t}+\boldsymbol{U} \cdot \nabla$ and $\overline{\boldsymbol{\omega}}=$ $\nabla \times\left(\boldsymbol{U}-q_{h}\left[\rho^{-1} n \mathbf{A}\right]\right)$, it is easy to see that the curl of equation (3.15) produces the generalized Ertel relation

$$
D_{t}(\overline{\boldsymbol{\omega}} \cdot \nabla \alpha)-(\overline{\boldsymbol{\omega}} \cdot \nabla) D_{t} \alpha=\nabla \alpha \cdot \nabla \times \mathbf{\Psi},
$$

where $\alpha$ is an arbitrary smooth function and $\Psi$ is the force expressed by (3.19). The quantity $\overline{\boldsymbol{\omega}} \cdot \nabla \alpha$ is the potential vorticity and the above relation generalizes Ertel's theorem to the current-coupling scheme of hybrid MHD.

Cross helicities. Upon denoting $\mathcal{V}=\boldsymbol{U}-q_{h} \rho^{-1} n \mathbf{A}$, the following two crosshelicities may now be defined:

$$
\Lambda_{1}=\int \boldsymbol{U} \cdot \mathbf{B} \mathrm{d}^{3} \mathbf{x} \quad \Lambda_{2}=\int \mathcal{V} \cdot \mathbf{B} \mathrm{d}^{3} \mathbf{x}
$$

However, while the first is conserved in time, i.e. $\mathrm{d} \Lambda_{1} / \mathrm{d} t=0$, the second satisfies

$$
\frac{\mathrm{d}}{\mathrm{d} t} \Lambda_{2}=-q_{h} \frac{\mathrm{d}}{\mathrm{d} t} \int \rho^{-1} n \mathbf{A} \cdot \mathbf{B} \mathrm{d}^{3} \mathbf{x}=q_{h} \int \rho^{-1}(\mathbf{A} \cdot \mathbf{B}) \nabla \cdot(\mathbf{K}-n \boldsymbol{U}) \mathrm{d}^{3} \mathbf{x},
$$

where the last non-vanishing integral is generated by the term parallel to $\mathbf{A}$ in equation (3.15).

\section{First pressure-coupling hybrid MHD scheme}

4.1. Formulation of the model. In this section we show how the variational structure of the previous current-coupling scheme provides a basis for the Euler-Poincaré formulation of a pressure-coupling scheme. This scheme establishes an equation for the total velocity

$$
\overline{\boldsymbol{U}}=\boldsymbol{U}+\frac{m_{h}}{\rho} \int \mathbf{v} f \mathrm{~d}^{3} \mathbf{v},
$$

under the assumption that the kinetic moment

$$
\mathbf{K}=\int \mathbf{v} f \mathrm{~d}^{3} \mathbf{v}
$$

does not contribute to the total energy of the system. This assumption can be justified if the energetic component is particularly rarefied, so that its density

$$
n=\int f \mathrm{~d}^{3} \mathbf{v}
$$

is negligible compared to the density $\rho$ of the cold fluid. This is precisely the hypothesis that we shall use in our derivation of the following energy-conserving pressure-coupling scheme obtained in [44]:

$$
\begin{aligned}
& \rho \frac{\partial \boldsymbol{U}}{\partial t}+\rho(\boldsymbol{U} \cdot \nabla) \boldsymbol{U}=-\nabla \mathbf{p}-m_{h} \nabla \cdot \int \mathbf{v v} f \mathrm{~d}^{3} \mathbf{v}-\frac{1}{\mu_{0}} \mathbf{B} \times \nabla \times \mathbf{B} \\
& \frac{\partial f}{\partial t}+(\boldsymbol{U}+\mathbf{v}) \cdot \frac{\partial f}{\partial \mathbf{x}}-\frac{\partial f}{\partial \mathbf{v}} \cdot \nabla \boldsymbol{U} \cdot \mathbf{v}+a_{h} \mathbf{v} \times \mathbf{B} \cdot \frac{\partial f}{\partial \mathbf{v}}=0 \\
& \frac{\partial \rho}{\partial t}+\nabla \cdot(\rho \boldsymbol{U})=0, \quad \frac{\partial \mathbf{B}}{\partial t}=\nabla \times(\boldsymbol{U} \times \mathbf{B}) .
\end{aligned}
$$


Here we have dropped the bar symbol for convenience. Before proceeding further, we remark that the $\boldsymbol{U}$-terms appearing in the kinetic equation (4.4) differ substantially from the corresponding term in the hybrid MHD model presented in [36] (whose Vlasov kinetic equation is replaced by its gyrokinetic approximation). Indeed, the fluid transport term $\boldsymbol{U} \cdot \partial_{\mathbf{x}} f$ is totally absent in reference [36], where the circulation force term $\partial_{\mathbf{v}} f \cdot \nabla \boldsymbol{U} \cdot \mathbf{v}$ is replaced by the Lorentz force $-q_{h} \boldsymbol{U} \times \mathbf{B}$ emerging in (3.8) as an electric field force (from ideal Ohm's law). More particularly, upon denoting $\mathbb{P}=\int \mathbf{v v} f \mathrm{~d}^{3} \mathbf{v}$, reference [36] derives a pressure-coupling scheme by assuming

$\partial_{t} \mathbf{K}=-\nabla \cdot \mathbb{P}+a_{h}(\mathbf{K}-n \boldsymbol{U}) \times \mathbf{B} \simeq 0$ in the current-coupling model (3.6)-(3.9); then the resulting force balance allows replacing Lorentz forces by the pressure term in the momentum equation (3.6). These crucial steps break the energy-conserving nature of the system, as explained in [44]. However, notice that the static equilibria of the above equations (4.3)-(4.5) coincide with those of the hybrid model in [36], provided the hot particles are governed by Vlasov dynamics.

4.2. Euler-Poincaré reduction by symmetry. Although the physical approximations leading to the pressure-coupling scheme present some problems that were summarized in [44], we shall see below how the variational approach to the model (4.3)-(4.5) produces an Euler-Poincaré system on a semidirect-product Lie group. At the reduced level, we shall prove that the Euler-Poincaré Lagrangian is a functional of the form

$$
l:\left(\mathfrak{X}\left(\mathbb{R}^{3}\right) \subseteq \mathfrak{X}\left(\mathbb{R}^{6}\right)\right) \times \operatorname{Den}\left(\mathbb{R}^{3}\right) \times \Omega^{1}\left(\mathbb{R}^{3}\right) \times \operatorname{Den}\left(\mathbb{R}^{6}\right) \rightarrow \mathbb{R},
$$

where the infinitesimal action that is involved in the semidirect-product Lie algebra $\mathfrak{X}\left(\mathbb{R}^{3}\right) \subseteq \mathfrak{X}\left(\mathbb{R}^{6}\right)$ is given by

$$
\boldsymbol{U} \cdot \mathbf{X}=£_{\mathbf{X}_{U}} \mathbf{X}, \quad \text { where } \quad \mathbf{X}_{U}:=(\boldsymbol{U},(\mathbf{v} \cdot \nabla) \boldsymbol{U}), \quad \forall \boldsymbol{U} \in \mathfrak{X}\left(\mathbb{R}^{3}\right) .
$$

This action naturally arises from the tangent-lifted action of $\operatorname{Diff}\left(\mathbb{R}^{3}\right)$ on $T \mathbb{R}^{3}=\mathbb{R}^{6}$, which in turn generates the natural $\operatorname{Diff}\left(\mathbb{R}^{3}\right)$-action on $\operatorname{Diff}\left(\mathbb{R}^{6}\right)$ (see Remark 4.3 below). On the other hand, the space of the advected quantities [21]

$$
(\rho, \mathbf{A}, f) \in \operatorname{Den}\left(\mathbb{R}^{3}\right) \times \Omega^{1}\left(\mathbb{R}^{3}\right) \times \operatorname{Den}\left(\mathbb{R}^{6}\right)
$$

involves the Lie algebra representation

$$
(\boldsymbol{U}, \mathbf{X}) \cdot(\rho, \mathbf{A}, f)=\left(£_{\boldsymbol{U}} \rho, £_{\boldsymbol{U}} \mathbf{A}, £_{\mathbf{X}+\mathbf{X}_{U}} f\right),
$$

whose associated diamond operation, defined by

$$
\left\langle\left(\frac{\delta l}{\delta \rho}, \frac{\delta l}{\delta \mathbf{A}}, \frac{\delta l}{\delta f}\right) \diamond(\rho, \mathbf{A}, f),(\boldsymbol{U}, \mathbf{X})\right\rangle:=-\left\langle\left(\frac{\delta l}{\delta \rho}, \frac{\delta l}{\delta \mathbf{A}}, \frac{\delta l}{\delta f}\right),\left(£_{\boldsymbol{U}} \rho, £_{\boldsymbol{U}} \mathbf{A}, £_{\mathbf{X}+\mathbf{X}_{U}} f\right)\right\rangle,
$$

will be derived explicitly in what follows.

At this point, the problem has been cast into the standard Euler-Poincaré theory for parameter-dependent Lagrangians $L: T G \times V^{*} \rightarrow \mathbb{R}$, with the peculiarity that the Lie group $G$ is a semidirect-product. Indeed, upon replacing $G$ by $G(H$, the pressure-coupling scheme will be written as an Euler-Poincaré variational principle on $T\left(G(S) \times V^{*}\right.$. Upon specializing to the case $G=\operatorname{Diff}\left(\mathbb{R}^{3}\right), H=\operatorname{Diff}\left(T \mathbb{R}^{3}\right)$, and $V^{*}=\operatorname{Den}\left(\mathbb{R}^{3}\right) \times \Omega^{1}\left(\mathbb{R}^{3}\right) \times \operatorname{Den}\left(\mathbb{R}^{6}\right)$, the Euler-Poincaré equations associated to such a 
Lagrangian can be written as follows on the reduced space $\mathfrak{X}\left(\mathbb{R}^{3}\right) \subseteq \mathfrak{X}\left(\mathbb{R}^{6}\right) \times V^{*}$ :

$$
\begin{aligned}
& \frac{\partial}{\partial t} \frac{\delta l}{\delta \boldsymbol{U}}+£_{\boldsymbol{U}} \frac{\delta l}{\delta \boldsymbol{U}}=\frac{\delta l}{\delta \mathbf{X}} \star \mathbf{X}+\left(\frac{\delta l}{\delta \rho}, \frac{\delta l}{\delta \mathbf{A}}, \frac{\delta l}{\delta f}\right) \diamond_{1}(\rho, \mathbf{A}, f), \\
& \frac{\partial \rho}{\partial t}+£_{\boldsymbol{U}} \rho=0, \quad \frac{\partial \mathbf{A}}{\partial t}+£_{\boldsymbol{U}} \mathbf{A}=0, \\
& \frac{\partial}{\partial t} \frac{\delta l}{\delta \mathbf{X}}+£_{\mathbf{X}+\mathbf{X}_{U}} \frac{\delta l}{\delta \mathbf{X}}=f \nabla_{(\mathbf{x}, \mathbf{v})} \frac{\delta l}{\delta f}, \\
& \frac{\partial f}{\partial t}+£_{\mathbf{X}+\mathbf{X}_{U}} f=0,
\end{aligned}
$$

where $\left(\diamond_{1}\right)$ in equation (4.7) denotes the $\boldsymbol{U}$-component of the diamond operation defined in (4.6), and the star $(\star)$ operation is defined as

$$
\left\langle\frac{\delta l}{\delta \mathbf{X}} \star \mathbf{X}, \boldsymbol{U}\right\rangle:=-\left\langle\frac{\delta l}{\delta \mathbf{X}}, £_{\mathbf{X}_{U}} \mathbf{X}\right\rangle .
$$

Integration by parts yields the more explicit expression,

$$
\begin{aligned}
\left\langle\frac{\delta l}{\delta \mathbf{X}} \star \mathbf{X}, \boldsymbol{U}\right\rangle & :=-\left\langle\frac{\delta l}{\delta \mathbf{X}}, £_{\mathbf{X}_{U}} \mathbf{X}\right\rangle \\
& =-\left\langle £_{\mathbf{X}} \frac{\delta l}{\delta \mathbf{X}},(\boldsymbol{U},(\mathbf{v} \cdot \nabla) \boldsymbol{U})\right\rangle \\
& =-\left\langle\int\left(£ \mathbf{X} \frac{\delta l}{\delta \mathbf{X}}\right)_{1} \mathrm{~d}^{3} \mathbf{v}-\nabla \cdot \int \mathbf{v}\left(£_{\mathbf{X}} \frac{\delta l}{\delta \mathbf{X}}\right)_{2} \mathrm{~d}^{3} \mathbf{v}, \boldsymbol{U}\right\rangle
\end{aligned}
$$

so that

$$
\frac{\delta l}{\delta \mathbf{X}} \star \mathbf{X}=-\int\left(£ \mathbf{X} \frac{\delta l}{\delta \mathbf{X}}\right)_{1} \mathrm{~d}^{3} \mathbf{v}+\nabla \cdot \int \mathbf{v}\left(£ \mathbf{X} \frac{\delta l}{\delta \mathbf{X}}\right)_{2} \mathrm{~d}^{3} \mathbf{v}
$$

where the indices 1 and 2 denote the $\mathbf{u}$ - and the a-components, respectively.

In order to complete the set of equations (4.7)-(4.10), we shall need a suitable Euler-Poincaré Lagrangian, which is given by

$$
\begin{aligned}
l(\boldsymbol{U}, \rho, \mathbf{X}, f, \mathbf{A})=\frac{1}{2} \int \rho|\boldsymbol{U}|^{2} & \mathrm{~d}^{3} \mathbf{x}-\int \rho \mathcal{U}(\rho) \mathrm{d}^{3} \mathbf{x}-\frac{1}{2 \mu_{0}} \int|\nabla \times \mathbf{A}|^{2} \mathrm{~d}^{3} \mathbf{x} \\
& +\int f\left(\frac{m_{h}}{2}|\mathbf{u}|^{2}+\frac{m_{h}}{2}|\mathbf{u}-\mathbf{v}|^{2}+q_{h} \mathbf{u} \cdot \mathbf{A}\right) \mathrm{d}^{3} \mathbf{x} \mathrm{d}^{3} \mathbf{v} .
\end{aligned}
$$

This Lagrangian is obtained from (3.10) by simply neglecting the term $q_{h} \int n \boldsymbol{U} \cdot \mathbf{A} \mathrm{d}^{3} \mathbf{x}$, consistently with the assumption of a rarefied energetic component.

The simplest starting point involves the kinetic part of the system (4.7)-(4.10), which is composed of the last two equations, i.e. (4.9)-(4.10). Let us start by calculating the functional derivatives. Upon using similar arguments as those in the previous section (and especially using the second component of equation (4.9)), in slightly different notation $(\mathbf{X}(\mathbf{x}, \mathbf{v})=(\mathbf{u}(\mathbf{x}, \mathbf{v}), \boldsymbol{\alpha}(\mathbf{x}, \mathbf{v})))$ one finds

$$
\mathbf{X}+\mathbf{X}_{\boldsymbol{U}}=(\mathbf{v}+\boldsymbol{U}, \boldsymbol{\alpha}+(\mathbf{v} \cdot \nabla) \boldsymbol{U}), \quad \frac{\delta l}{\delta \mathbf{u}}=m_{h} f\left(\mathbf{v}+a_{h} \mathbf{A}\right), \quad \frac{\delta l}{\delta f}=\frac{m_{h}}{2}|\mathbf{v}|^{2}+q_{h} \mathbf{v} \cdot \mathbf{A}
$$


Upon denoting $\boldsymbol{p}(\mathbf{x}, \mathbf{v})=m_{h} \mathbf{v}+q_{h} \mathbf{A}(\mathbf{x})$, we divide equation (2.10) by $f$, so that

$$
\frac{\partial}{\partial t}\left(\frac{1}{f} \frac{\delta l}{\delta \mathbf{X}}\right)+£ \mathbf{X}+\mathbf{X}_{U}\left(\frac{1}{f} \frac{\delta l}{\delta \mathbf{X}}\right)=\nabla_{(\mathbf{x}, \mathbf{v})} \frac{\delta l}{\delta f},
$$

and project it onto the first component to obtain

$$
\partial_{t} \boldsymbol{p}+\left((\mathbf{v}+\boldsymbol{U}) \cdot \partial_{\mathbf{x}}\right) \boldsymbol{p}+\left(\boldsymbol{\alpha} \cdot \partial_{\mathbf{v}}\right) \boldsymbol{p}+\left(\left(\mathbf{v} \cdot \partial_{\mathbf{x}}\right) \boldsymbol{U} \cdot \partial_{\mathbf{v}}\right) \boldsymbol{p}+\partial_{\mathbf{x}}(\mathbf{v}+\boldsymbol{U}) \cdot \boldsymbol{p}=\partial_{\mathbf{x}}\left(\frac{m_{h}}{2}|\mathbf{v}|^{2}+q_{h} \mathbf{v} \cdot \mathbf{A}\right) .
$$

Then, upon using the second equation in (4.8) as well as standard vector identities, one writes

$$
\boldsymbol{\alpha}=a_{h} \mathbf{v} \times(\nabla \times \mathbf{A})-\nabla \boldsymbol{U} \cdot \mathbf{v}-(\mathbf{v} \cdot \nabla) \boldsymbol{U}
$$

and the vector field $\mathbf{X}+\mathbf{X}_{U}$ becomes

$$
\mathbf{X}+\mathbf{X}_{\boldsymbol{U}}=\left(\mathbf{v}+\boldsymbol{U}, a_{h} \mathbf{v} \times(\nabla \times \mathbf{A})-\nabla \boldsymbol{U} \cdot \mathbf{v}\right) .
$$

In turn, upon noticing that $\nabla_{(\mathbf{x}, \mathbf{v})} \cdot\left(\mathbf{X}+\mathbf{X}_{U}\right)=0$, this vector field produces the Vlasov kinetic equation (4.9) in the form

$$
\frac{\partial f}{\partial t}+(\mathbf{v}+\boldsymbol{U}) \cdot \frac{\partial f}{\partial \mathbf{x}}-\left(\nabla \boldsymbol{U} \cdot \mathbf{v}-a_{h} \mathbf{v} \times(\nabla \times \mathbf{A})\right) \cdot \frac{\partial f}{\partial \mathbf{v}}=0
$$

which is identical to (4.4).

At this point, one needs to verify that equation (4.7) effectively returns the velocity equation (4.3) of the pressure-coupling scheme (4.3)-(4.5). To this purpose, we shall use the following

Lemma 4.1. In the special case when

$$
\mathbf{X}(\mathbf{x}, \mathbf{v})=(\mathbf{v}, \boldsymbol{\alpha}(\mathbf{x}, \mathbf{v})) \quad \text { and } \quad \frac{\delta l}{\delta \mathbf{X}}(\mathbf{x}, \mathbf{v})=(\mathbf{w}(\mathbf{x}, \mathbf{v}), 0),
$$

then

$$
\mathbf{X} \star \frac{\delta l}{\delta \mathbf{X}}=0
$$

for arbitrary vector quantities $\boldsymbol{\alpha}(\mathbf{x}, \mathbf{v})$ and $\mathbf{w}(\mathbf{x}, \mathbf{v})$.

Proof. The proof follows by direct verification, upon writing the definition of the star operation in (4.11) as

$$
\begin{aligned}
\left\langle\mathbf{X} \star \frac{\delta l}{\delta \mathbf{X}}, \boldsymbol{U}\right\rangle:=\left\langle\frac{\delta l}{\delta \mathbf{X}},\left[\mathbf{X}_{\boldsymbol{U}}, \mathbf{X}\right]\right\rangle & =\left\langle\frac{\delta l}{\delta \mathbf{X}},\left(\left(\mathbf{X}_{\boldsymbol{U}} \cdot \nabla_{(\mathbf{x}, \mathbf{v})}\right) \mathbf{X}-\left(\mathbf{X} \cdot \nabla_{(\mathbf{x}, \mathbf{v})}\right) \mathbf{X}_{\boldsymbol{U}}\right)\right\rangle \\
& =\left\langle\mathbf{w},\left(\left(\mathbf{X}_{\boldsymbol{U}} \cdot \nabla_{(\mathbf{x}, \mathbf{v})}\right) \mathbf{v}-\left(\mathbf{X} \cdot \nabla_{(\mathbf{x}, \mathbf{v})}\right) \boldsymbol{U}\right)\right\rangle \\
& =\left\langle\mathbf{w},\left(\left((\mathbf{v} \cdot \nabla) \boldsymbol{U} \cdot \partial_{\mathbf{v}}\right) \mathbf{v}-(\mathbf{v} \cdot \nabla) \boldsymbol{U}\right)\right\rangle \\
& =\langle\mathbf{w},((\mathbf{v} \cdot \nabla) \boldsymbol{U}-(\mathbf{v} \cdot \nabla) \boldsymbol{U})\rangle=0,
\end{aligned}
$$

in which the last step uses integration by parts of the first term. 
Another result that we shall need is the following formula for the diamond operation in (4.11):

$$
\frac{\delta l}{\delta f} \diamond_{1} f=\int f \partial_{\mathbf{x}} \frac{\delta l}{\delta f} \mathrm{~d}^{3} \mathbf{v}-\nabla \cdot \int f \mathbf{v} \partial_{\mathbf{v}} \frac{\delta l}{\delta f} \mathrm{~d}^{3} \mathbf{v},
$$

which may be directly verified from its definition

$$
\left\langle\frac{\delta l}{\delta f} \diamond_{1} f, \boldsymbol{U}\right\rangle:=\left\langle f,\left(\mathbf{X}_{U} \cdot \nabla_{(\mathbf{x}, \mathbf{v})}\right) \frac{\delta l}{\delta f}\right\rangle .
$$

Now, upon recalling the particular form of the variational derivative

$$
\frac{\delta l}{\delta f}=\frac{1}{2} m_{h}|\mathbf{v}|^{2}+q_{h} \mathbf{v} \cdot \mathbf{A},
$$

we calculate

$$
\begin{aligned}
\frac{\delta l}{\delta f} \diamond_{1} f & =q_{h} \nabla \mathbf{A} \cdot \mathbf{K}-m_{h} \nabla \cdot \mathbb{P}-q_{h}(\nabla \cdot \mathbf{K}) \mathbf{A}-q_{h}(\mathbf{K} \cdot \nabla) \mathbf{A} \\
& =q_{h} \mathbf{K} \times \mathbf{B}-q_{h}(\nabla \cdot \mathbf{K}) \mathbf{A}-m_{h} \nabla \cdot \mathbb{P},
\end{aligned}
$$

where we recall the definition (4.1) of the averaged kinetic momentum and we have introduced the absolute pressure tensor

$$
\mathbb{P}=\int \mathbf{v v} f \mathrm{~d}^{3} \mathbf{v}
$$

Then, upon writing

$$
\frac{\delta l}{\delta \rho} \diamond_{1} \rho=\rho \nabla \frac{\delta l}{\delta \rho}, \quad \frac{\delta l}{\delta \mathbf{A}} \diamond_{1} \mathbf{A}=-\frac{\delta l}{\delta \mathbf{A}} \times \nabla \times \mathbf{A}+\left(\nabla \cdot \frac{\delta l}{\delta \mathbf{A}}\right) \mathbf{A}
$$

and evaluating

$$
\frac{\delta l}{\delta \boldsymbol{U}}=\rho \boldsymbol{U}, \quad \frac{\delta l}{\delta \mathbf{A}}=-\nabla \times \nabla \times \mathbf{A}+q_{h} \mathbf{K}, \quad \frac{\delta l}{\delta \rho}=\frac{1}{2}|\boldsymbol{U}|^{2}+\rho \mathcal{U}^{\prime}(\rho)+\mathcal{U}(\rho),
$$

we see that equation (4.7) returns the velocity equation (4.3) of the pressure-coupling scheme (4.3)-(4.5). In conclusion, we have proven the following theorem.

THEOREM 4.2. The hybrid pressure-coupling MHD scheme (4.3)-(4.5) arises from the Euler-Poincaré variational principle

$$
\delta \int_{t_{0}}^{t_{1}} l(\boldsymbol{U}, \rho, \mathbf{X}, f, \mathbf{A}) \mathrm{d} t=0
$$

with the reduced Lagrangian

$$
l:\left(\mathfrak{X}\left(\mathbb{R}^{3}\right) \subseteq \mathfrak{X}\left(\mathbb{R}^{6}\right)\right) \times C^{\infty}\left(\mathbb{R}^{3}\right)^{*} \times \Omega^{1}\left(\mathbb{R}^{3}\right) \times C^{\infty}\left(\mathbb{R}^{6}\right)^{*} \rightarrow \mathbb{R},
$$

given in (4.13), and variations

$$
\begin{aligned}
& \delta(\boldsymbol{U}, \mathbf{X})=\partial_{t}(\mathbf{W}, \mathbf{Z})-\left(£_{\boldsymbol{U}} \mathbf{W}, £_{\mathbf{X}_{\mathbf{W}}} \mathbf{X}-£_{\mathbf{X}_{U}} \mathbf{Z}+£_{\mathbf{X}} \mathbf{Z}\right), \\
& \delta f=-£_{\mathbf{Z}+\mathbf{X}_{\mathbf{W}}} f, \quad \delta(\rho, \mathbf{A})=-£_{\mathbf{W}}(\rho, \mathbf{A}),
\end{aligned}
$$


where the vector fields $\mathbf{W} \in \mathfrak{X}\left(\mathbb{R}^{3}\right)$ and $\mathbf{Z} \in \mathfrak{X}\left(\mathbb{R}^{6}\right)$ vanish at the endpoints. This variational principle is equivalent to the Euler-Poincaré equations (4.7)-(4.10), which hold for an arbitrary hybrid Lagrangian.

REMARK 4.3 (Conjugation action in semidirect-product Lie groups). The Lie algebra action that is involved in the semidirect product $\mathfrak{X}\left(\mathbb{R}^{3}\right)\left(\mathfrak{X}\left(\mathbb{R}^{6}\right)\right.$ is naturally inherited from the Jacobi-Lie bracket on $\mathfrak{X}\left(\mathbb{R}^{6}\right)$. According to the theory of semidirectproduct Lie groups, this action must arise from a group action of $\operatorname{Diff}\left(\mathbb{R}^{3}\right)$ on $\operatorname{Diff}\left(\mathbb{R}^{6}\right)$ that is also a group homomorphism. In other words, $\eta\left(\psi_{1} \psi_{2}\right)=\eta\left(\psi_{1}\right) \eta\left(\psi_{2}\right)$, with $\eta \in \operatorname{Diff}\left(\mathbb{R}^{3}\right)$ and $\psi_{1}, \psi_{2} \in \operatorname{Diff}\left(\mathbb{R}^{6}\right)$. In particular, since we can regard $\operatorname{Diff}\left(\mathbb{R}^{3}\right)$ as a subgroup of $\operatorname{Diff}\left(\mathbb{R}^{6}\right)$, one is led to consider the action $\psi \mapsto \eta \circ \psi \circ \eta^{-1}$ which is naturally inherited from the conjugation action in $\operatorname{Diff}\left(\mathbb{R}^{6}\right)$. This action generates the semidirect-product Lie group $\operatorname{Diff}\left(\mathbb{R}^{3}\right)\left(\operatorname{Diff}\left(\mathbb{R}^{6}\right)\right.$, whose tangent space at the identity $\mathfrak{X}\left(\mathbb{R}^{3}\right) \subseteq \mathfrak{X}\left(\mathbb{R}^{6}\right)$ is endowed with the Lie bracket

$$
[(\boldsymbol{U}, \mathbf{X}),(\boldsymbol{W}, \mathbf{Z})]=-\left(£_{\boldsymbol{U}} \mathbf{W}, £_{\mathbf{X}_{\mathbf{W}}} \mathbf{X}-£_{\mathbf{X}_{U}} \mathbf{Z}+£_{\mathbf{X}} \mathbf{Z}\right) .
$$

More details on semidirect-products of two Lie groups may be found in [28, 4].

4.3. Discussion. The Euler-Poincaré construction of the first pressure coupling scheme is based on the following proposition:

Proposition 4.4. The Euler-Poincaré equations (4.3)-(4.5) yield

$$
\begin{aligned}
& \left(\frac{\partial}{\partial t}+£_{\boldsymbol{U}}\right)\left(\frac{\delta l}{\delta \boldsymbol{U}}-\int \frac{\delta l}{\delta \mathbf{u}} \mathrm{d}^{3} \mathbf{v}+\int\left(\mathbf{v} \cdot \partial_{\mathbf{x}}\right) \frac{\delta l}{\delta \boldsymbol{\alpha}} \mathrm{d}^{3} \mathbf{v}\right) \\
= & \rho \nabla \frac{\delta l}{\delta \rho}-\frac{\delta l}{\delta \mathbf{A}} \times \nabla \times \mathbf{A}+\left(\nabla \cdot \frac{\delta l}{\delta \mathbf{A}}\right) \mathbf{A} .
\end{aligned}
$$

Proof. The proof is a direct verification, based on relations (4.12) and (4.14). After computing

$$
\begin{aligned}
& \left(\frac{\partial}{\partial t}+£_{\boldsymbol{U}}\right)\left(\frac{\delta l}{\delta \boldsymbol{U}}-\int \frac{\delta l}{\delta \mathbf{u}} \mathrm{d}^{3} \mathbf{v}+\int\left(\mathbf{v} \cdot \partial_{\mathbf{x}}\right) \frac{\delta l}{\delta \boldsymbol{\alpha}} \mathrm{d}^{3} \mathbf{v}\right) \\
= & \frac{\delta l}{\delta \rho} \diamond_{1} \rho+\frac{\delta l}{\delta \mathbf{A}} \diamond_{1} \mathbf{A}+\int\left(£_{\mathbf{X}_{U}} \frac{\delta l}{\delta \mathbf{X}}\right)_{1} \mathrm{~d}^{3} \mathbf{v}-£_{\boldsymbol{U}} \int \frac{\delta l}{\delta \mathbf{u}} \mathrm{d}^{3} \mathbf{v} \\
& -\int\left(\mathbf{v} \cdot \partial_{\mathbf{x}}\right)\left(£_{\mathbf{X}_{U}} \frac{\delta l}{\delta \mathbf{X}}\right)_{2} \mathrm{~d}^{3} \mathbf{v}+£_{\boldsymbol{U}} \int\left(\mathbf{v} \cdot \partial_{\mathbf{x}}\right) \frac{\delta l}{\delta \boldsymbol{\alpha}} \mathrm{d}^{3} \mathbf{v},
\end{aligned}
$$

the proof follows immediately from Lemma 4.5 below.

Kelvin-Noether theorem and its momentum map. The above relation represents the Lagrangian analogue of an important construction in Lie-Poisson Hamiltonian systems, known as untangling. Untangling is accomplished by shifting the momentum by a momentum map that takes the Lie-Poisson bracket on the dual of a semidirect-product Lie algebra into the Lie-Poisson bracket on the dual of a direct-sum Lie algebra. For more details, see Corollary 2.4 in [24].

It is perhaps not surprising that the very first application of this construction occurred in plasma physics $[18,17]$. This construction was also used in [44]. The momentum map in the present case is the dual $i^{*}: \mathfrak{X}^{*}\left(\mathbb{R}^{6}\right) \rightarrow \mathfrak{X}^{*}\left(\mathbb{R}^{3}\right)$ of the Lie algebra inclusion $i: \boldsymbol{U} \mapsto \mathbf{X}_{\boldsymbol{U}}$. The result (4.15) hinges on the following property, which is proven in Appendix A: 
Lemma 4.5. The following map $i^{*}: \mathfrak{X}^{*}\left(\mathbb{R}^{6}\right) \rightarrow \mathfrak{X}^{*}\left(\mathbb{R}^{3}\right)$ :

$$
i^{*}\left(\frac{\delta l}{\delta \mathbf{X}}\right)=\int \frac{\delta l}{\delta \mathbf{u}} \mathrm{d}^{3} \mathbf{v}-\int\left(\mathbf{v} \cdot \partial_{\mathbf{x}}\right) \frac{\delta l}{\delta \boldsymbol{\alpha}} \mathrm{d}^{3} \mathbf{v},
$$

is a momentum map satisfying the relation

$$
i^{*}\left(£_{\mathbf{X}_{U}} \frac{\delta l}{\delta \mathbf{X}}\right)=£_{U} i^{*}\left(\frac{\delta l}{\delta \mathbf{X}}\right)
$$

for an arbitrary vector field $\boldsymbol{U} \in \mathfrak{X}\left(\mathbb{R}^{3}\right)$.

Notice that the momentum map $i^{*}: \mathfrak{X}^{*}\left(\mathbb{R}^{6}\right) \rightarrow \mathfrak{X}^{*}\left(\mathbb{R}^{3}\right)$ is different in nature from the star operator $\star: \mathfrak{X}\left(\mathbb{R}^{6}\right) \times \mathfrak{X}^{*}\left(\mathbb{R}^{6}\right) \rightarrow \mathfrak{X}^{*}\left(\mathbb{R}^{3}\right)$ introduced in (4.11). Indeed, while the latter arises from the cotangent lift of the $\operatorname{Diff}\left(\mathbb{R}^{3}\right)$-action on $\mathfrak{X}\left(\mathbb{R}^{6}\right)$, the momentum map $i^{*}$ arises from the $\operatorname{Diff}\left(\mathbb{R}^{3}\right)$-action on $\operatorname{Diff}\left(\mathbb{R}^{6}\right)$, which is given by conjugation, as explained in Remark 4.3. The momentum map property of $i^{*}$ can be easily verified since the inclusion $i: \mathfrak{X}\left(\mathbb{R}^{3}\right) \hookrightarrow \mathfrak{X}\left(\mathbb{R}^{6}\right)$ is the dual of a Lie algebra homomorphism, i.e.

$$
\left[\mathbf{X}_{\boldsymbol{U}}, \mathbf{X}_{\boldsymbol{W}}\right]=[i(\boldsymbol{U}), i(\boldsymbol{W})]=i([\boldsymbol{U}, \boldsymbol{W}])=\mathbf{X}_{[\boldsymbol{U}, \boldsymbol{W}]},
$$

where $[\cdot, \cdot]$ denotes minus the Jacobi-Lie bracket on $\mathfrak{X}\left(\mathbb{R}^{3}\right)$ or $\mathfrak{X}\left(\mathbb{R}^{6}\right)$, depending on the context. The proof in Appendix A shows explicitly that $i^{*}$ satisfies the definition of momentum map.

An immediate consequence of Proposition 4.4 is the following circulation law for the hybrid scheme (4.3)-(4.5), which recovers the previous results in [44].

COROLlary 4.6 (Kelvin circulation law). The pressure-coupling MHD scheme (4.3)-(4.5) possesses the following equivalent circulation theorems:

$$
\begin{aligned}
& \frac{\mathrm{d}}{\mathrm{d} t} \oint_{\gamma_{t}(\boldsymbol{U})} \boldsymbol{U} \cdot \mathrm{d} \mathbf{x}=-\oint_{\gamma_{t}(\boldsymbol{U})} \frac{1}{\rho}\left(\frac{1}{\mu_{0}} \mathbf{B} \times \nabla \times \mathbf{B}+m_{h} \nabla \cdot \int \mathbf{v} \mathbf{v} f \mathrm{~d}^{3} \mathbf{v}\right) \cdot \mathrm{d} \mathbf{x}, \\
& \frac{\mathrm{d}}{\mathrm{d} t} \oint_{\gamma_{t}(\boldsymbol{U})}\left(\boldsymbol{U}-\frac{1}{\rho} \int f \boldsymbol{p} \mathrm{d}^{3} \mathbf{v}\right) \cdot \mathrm{d} \mathbf{x} \\
= & -\oint_{\gamma_{t}(\boldsymbol{U})} \frac{1}{\rho}\left(\mathbf{B} \times\left(\mu_{0}^{-1} \nabla \times \mathbf{B}-q_{h} \mathbf{K}\right)-q_{h}(\nabla \cdot \mathbf{K}) \mathbf{A}\right) \cdot \mathrm{d} \mathbf{x} .
\end{aligned}
$$

Proof. Upon considering the Euler-Poincaré Lagrangian (4.13), relation (4.16) is implied by the Euler-Poincaré theorem; see [21] and the equation of motion (4.7). On the other hand, relation (4.17) is an immediate consequence of equation (4.15) in Proposition 4.4.

Notice that taking the difference of the above circulation laws yields

$$
\frac{\mathrm{d}}{\mathrm{d} t} \oint_{\gamma_{t}(\boldsymbol{U})}\left(\frac{1}{\rho} \int f \boldsymbol{p} \mathrm{d}^{3} \mathbf{v}\right) \cdot \mathrm{d} \mathbf{x}=-\oint_{\gamma_{t}(\boldsymbol{U})} \frac{1}{\rho}\left(m_{h} \nabla \cdot \int \mathbf{v} \mathbf{v} f \mathrm{~d}^{3} \mathbf{v}-q_{h} \mathbf{K} \times \mathbf{B}+q_{h}(\nabla \cdot \mathbf{K}) \mathbf{A}\right) \cdot \mathrm{d} \mathbf{x},
$$

where we recall the relation $\boldsymbol{p}=m_{h} \mathbf{v}+q_{h} \mathbf{A}$. Thus, upon considering (4.9) and the zero-th moment equation $\partial_{t} n+\nabla \cdot(n \boldsymbol{U})=-\nabla \cdot \mathbf{K}$ associated to (4.4), we have

$$
\frac{\mathrm{d}}{\mathrm{d} t} \oint_{\gamma_{t}(\boldsymbol{U})} \frac{\mathbf{K}}{\rho} \cdot \mathrm{d} \mathbf{x}=-\oint_{\gamma_{t}(\boldsymbol{U})} \frac{1}{\rho}\left(\nabla \cdot \int \mathbf{v} \mathbf{v} f \mathrm{~d}^{3} \mathbf{v}-a_{h} \mathbf{K} \times \mathbf{B}\right) \cdot \mathrm{d} \mathbf{x} .
$$


Moreover, the above fluid circulation laws are accompanied by preservation of the Poincaré-invariant

$$
\frac{\mathrm{d}}{\mathrm{d} t} \oint_{\zeta_{t}\left(\mathbf{X}+\mathbf{X}_{U}\right)} \boldsymbol{p} \cdot \mathrm{d} \mathbf{x}=0
$$

where the curve $\zeta_{t}$ now moves along the total phase-space vector field $\mathbf{X}+\mathbf{X}_{U}$.

Ertel's theorem. By proceeding as in the corresponding treatment for the currentcoupling scheme, one recognizes that the force

$$
\mathbf{\Psi}=\left(\mu_{0}^{-1} \nabla \times \mathbf{B}-q_{h} \mathbf{K}\right) \times \mathbf{B}+q_{h}(\nabla \cdot \mathbf{K}) \mathbf{A}
$$

provides the opportunity to generalize Ertel's relation for MHD [15] to apply to the first pressure-coupling scheme. Indeed, upon following similar steps as those in Section 3.3, one finds that the incompressible form of equation (4.15) yields the relation (3.20), with $\overline{\boldsymbol{\omega}}=\nabla \times\left(\boldsymbol{U}-\rho^{-1} \int f \boldsymbol{p} \mathrm{d}^{3} \mathbf{v}\right)$ and $\boldsymbol{\Psi}$ as given in (4.18). Again, we kept the density $\rho$ in the expression of $\overline{\boldsymbol{\omega}}$ in order to provide correct dimensions; incompressible flows are always accompanied by $\rho=1$.

Cross helicities. Notice that, upon denoting $\mathcal{W}=\boldsymbol{U}-\rho^{-1} \int f \boldsymbol{p} \mathrm{d}^{3} \mathbf{v}$, both of the cross helicities

$$
\Lambda_{1}=\int \boldsymbol{U} \cdot \mathbf{B} \mathrm{d}^{3} \mathbf{x}, \quad \Lambda_{3}=\int \mathcal{W} \cdot \mathbf{B} \mathrm{d}^{3} \mathbf{x}
$$

possess nontrivial dynamics. Indeed, their equations of motion read as

$$
\frac{\mathrm{d} \Lambda_{1}}{\mathrm{~d} t}=-m_{h} \int \rho^{-1}(\nabla \cdot \mathbb{P}) \cdot \mathbf{B} \mathrm{d}^{3} \mathbf{x}, \quad \frac{\mathrm{d} \Lambda_{3}}{\mathrm{~d} t}=q_{h} \int \rho^{-1}(\mathbf{A} \cdot \mathbf{B}) \nabla \cdot \mathbf{K} \mathrm{d}^{3} \mathbf{x} .
$$

On the other hand, the following cross helicity is conserved:

$$
\Lambda_{2}=\int \Upsilon \cdot \mathbf{B} \mathrm{d}^{3} \mathbf{x}
$$

where we have denoted $\mathbf{\Upsilon}=\boldsymbol{U}-m_{h} \rho^{-1} \mathbf{K}$. Upon noticing that $D_{t}\left(\rho^{-1} n\right)=$ $\rho^{-1}\left(\partial_{t} n+\nabla \cdot(n \boldsymbol{U})\right)$, the conservation of $\Lambda_{2}$ is readily seen by computing

$$
\frac{\mathrm{d} \Lambda_{2}}{\mathrm{~d} t}=\frac{\mathrm{d} \Lambda_{3}}{\mathrm{~d} t}+q_{h} \frac{\mathrm{d}}{\mathrm{d} t} \int \rho^{-1} n \mathbf{A} \cdot \mathbf{B} \mathrm{d}^{3} \mathbf{x}=0,
$$

where one considers the equation $\partial_{t} n+\nabla \cdot(n \boldsymbol{U})=-\nabla \cdot \mathbf{K}$ arising from the zeroth moment of the Vlasov equation (4.4).

\section{Second pressure-coupling hybrid MHD scheme}

5.1. Formulation of the model. As mentioned in the previous section, the pressure-coupling MHD scheme is conventionally obtained under the assumption that the hot plasma component is rarefied. Upon denoting by $n$ the particle density of the hot component and by $m_{c}$ the cold particle mass, this assumption reads as $n \ll \rho / m_{c}$. Then, in order to avoid divergences in the mean velocity $\boldsymbol{V}=n^{-1} \int \mathbf{v} f \mathrm{~d}^{3} \mathbf{v}$ of the hot component, a small hot particle density $n$ requires the hot momentum $\mathbf{K}=\int \mathbf{v} f \mathrm{~d}^{3} \mathbf{v}$ to also be small. Thus, it is customary to replace the total momentum $\rho \boldsymbol{U}+m_{h} \mathbf{K}$ by simply $\rho \boldsymbol{U}$, i.e. the cold fluid momentum. While this operation is often performed on 
the equations of motion $[8,36]$, our approach makes this replacement directly in the variational principle, resulting in agreement with [44]. The advantage of modeling in the Lagrangian of the Euler-Poincaré variational principle is that it always produces circulation theorems. This is the content of the Kelvin-Noether theorem of [21].

The assumption of a rarefied hot component may also require that the mean kinetic energy $\frac{m_{h}}{2} \int n|\boldsymbol{V}|^{2} \mathrm{~d}^{3} \mathbf{x}$ of the hot component is subtracted from the corresponding total kinetic energy $\frac{m_{h}}{2} \int f|\mathbf{v}|^{2} \mathrm{~d}^{3} \mathbf{x} \mathrm{d}^{3} \mathbf{v}$. This operation yields the second pressure-coupling scheme [44]

$$
\begin{aligned}
& \rho \frac{\partial \boldsymbol{U}}{\partial t}+\rho(\boldsymbol{U} \cdot \nabla) \boldsymbol{U}=-\nabla \mathbf{p}-m_{h} \nabla \cdot \int\left(\mathbf{v}-\frac{\mathbf{K}}{n}\right)\left(\mathbf{v}-\frac{\mathbf{K}}{n}\right) f \mathrm{~d}^{3} \mathbf{v}-\frac{1}{\mu_{0}} \mathbf{B} \times \nabla \times \mathbf{B}, \\
& \frac{\partial f}{\partial t}+\left(\mathbf{v}+\boldsymbol{U}-\frac{\mathbf{K}}{n}\right) \cdot \frac{\partial f}{\partial \mathbf{x}}+\left(a_{h}\left(\mathbf{v}-\frac{\mathbf{K}}{n}\right) \times \mathbf{B}-\nabla \boldsymbol{U} \cdot \mathbf{v}+\left(\nabla \frac{\mathbf{K}}{n}\right) \cdot\left(\mathbf{v}-\frac{\mathbf{K}}{n}\right)\right) \cdot \frac{\partial f}{\partial \mathbf{v}}=0 \\
& \frac{\partial \rho}{\partial t}+\nabla \cdot(\rho \boldsymbol{U})=0, \quad \frac{\partial \mathbf{B}}{\partial t}=\nabla \times(\boldsymbol{U} \times \mathbf{B}) .
\end{aligned}
$$

We remark that neglecting all $\boldsymbol{U}$ - and $n^{-1} \mathbf{K}$-terms in the kinetic equation (5.2) and replacing $n^{-1} \mathbf{K} \times \mathbf{B}$ by $\boldsymbol{U} \times \mathbf{B}$ produces the hybrid MHD model in [23, 43] (although the general Vlasov equation is adopted here, rather than a drift-kinetic equation). Upon denoting $\overline{\mathbb{P}}=\int(\mathbf{v}-\langle\mathbf{v}\rangle)^{\otimes 2} f \mathrm{~d}^{3} \mathbf{v}$ and $\langle\mathbf{v}\rangle=\mathbf{K} / n$, the model in [23, 43] can be derived by assuming $n \partial_{t}\langle\mathbf{v}\rangle+n(\langle\mathbf{v}\rangle \cdot \nabla)\langle\mathbf{v}\rangle=-\nabla \cdot \overline{\mathbb{P}}+a_{h}(\mathbf{K}-n \boldsymbol{U}) \times \mathbf{B} \simeq 0$ in the current-coupling scheme (3.6)-(3.9), so that the Lorentz forces in (3.6) are replaced by a relative pressure term.

Notice that the static equilibria of the equations (5.1)-(5.3) coincide with those of the hybrid model in [23, 43] (for hot particles undergoing Vlasov dynamics), provided the equilibrium Vlasov distribution (usually denoted by $f_{0}$ ) is isotropic in the velocity coordinate, i.e. $\mathbf{K}_{0}=\int \mathbf{v} f_{0} \mathrm{~d}^{3} \mathbf{v}=0$.

5.2. Euler-Poincaré reduction by symmetry. In order to obtain the Euler-Poincaré formulation of the hybrid model (5.1)-(5.3) for the second pressurecoupling scheme [44], the Lagrangian (4.13) is transformed into

$$
\begin{aligned}
& l(\boldsymbol{U}, \boldsymbol{V}, \mathbf{X}, \rho, \mathbf{A}, f) \\
= & \frac{1}{2} \int \rho|\boldsymbol{U}|^{2} \mathrm{~d}^{3} \mathbf{x}-\int \rho \mathcal{U}(\rho) \mathrm{d}^{3} \mathbf{x}-\frac{1}{2 \mu_{0}} \int|\nabla \times \mathbf{A}|^{2} \mathrm{~d}^{3} \mathbf{x} \\
& +\int f\left(\frac{m_{h}}{2}|\mathbf{u}|^{2}+\frac{m_{h}}{2}|\mathbf{u}-\mathbf{v}|^{2}-\frac{m_{h}}{2}|\boldsymbol{V}|^{2}+q_{h}(\mathbf{u}+\boldsymbol{V}) \cdot \mathbf{A}\right) \mathrm{d}^{3} \mathbf{x} \mathrm{d}^{3} \mathbf{v},
\end{aligned}
$$

where we notice that the mean velocity $\boldsymbol{V}$ appears as a new dynamical variable. The term $q_{h} \int f \boldsymbol{V} \cdot \mathbf{A} \mathrm{d}^{3} \mathbf{x d}^{3} \mathbf{v}$ has been inserted in order to match the correct Lorentz force on the hot component [44]. As we shall see, the two $\boldsymbol{V}$-terms in the above Lagrangian correspond to subtracting the contributions of the mean velocity $\langle\mathbf{v}\rangle=n^{-1} \mathbf{K}=-\boldsymbol{V}$. Upon following the same reasoning as in the previous section, we realize that the fluid $\boldsymbol{U}$-transport exerted by the cold fluid component on the Vlasov distribution $f$ of the hot particles must imply a $\boldsymbol{U}$-transport of the mean hot velocity $\boldsymbol{V}$. More particularly, we interpret the above Lagrangian as a functional of the type

$$
l: \mathfrak{X}_{1}\left(\mathbb{R}^{3}\right) \subseteq\left(\mathfrak{X}_{2}\left(\mathbb{R}^{3}\right) \subseteq \mathfrak{X}\left(\mathbb{R}^{6}\right)\right) \times \operatorname{Den}\left(\mathbb{R}^{3}\right) \times \Omega^{1}\left(\mathbb{R}^{3}\right) \times \operatorname{Den}\left(\mathbb{R}^{6}\right) \rightarrow \mathbb{R},
$$

where $\mathfrak{X}_{1}\left(\mathbb{R}^{3}\right)$ and $\mathfrak{X}_{2}\left(\mathbb{R}^{3}\right)$ are two copies of the same Lie algebra $\mathfrak{X}\left(\mathbb{R}^{3}\right)$ of vector fields, although they are denoted differently because the second is assumed to act 
trivially on the space Den $\left(\mathbb{R}^{3}\right) \times \Omega^{1}\left(\mathbb{R}^{3}\right)$ containing the cold fluid density $\rho$ as well as the magnetic potential A. The first (outer) semidirect-product symbol corresponds to fluid $\boldsymbol{U}$-transport of both the mean velocity $\boldsymbol{V} \in \mathfrak{X}_{2}\left(\mathbb{R}^{3}\right)$ and the phase-space vector field $\mathbf{X} \in \mathfrak{X}\left(\mathbb{R}^{6}\right)$. On the other hand, the second (inner) semidirect-product symbol corresponds to the $\boldsymbol{V}$-transport exerted by the mean flow of the hot component on its corresponding phase-space velocity. At the group level, the unreduced Lagrangian is of the type

$$
L_{\rho_{0}, \mathbf{A}_{0}, f_{0}}: T\left(\operatorname{Diff}_{1}\left(\mathbb{R}^{3}\right) \subseteq\left(\operatorname{Diff}_{2}\left(\mathbb{R}^{3}\right) \subseteq \operatorname{Diff}\left(\mathbb{R}^{6}\right)\right)\right) \rightarrow \mathbb{R},
$$

where $\left(\rho_{0}, \mathbf{A}_{0}, f_{0}\right)$ are the advected parameters. Notice that similar arguments to those in Remark 4.3 also apply here about the group actions involved in nested semidirectproduct Lie group structures of this kind. The first instance of nested semidirectproduct Lie-group structures also occurred in plasma physics - in the discovery of the Lie-Poisson brackets dual to nested semidirect-product Lie algebras in models of Alfvén wave turbulence $[14,16]$. This construction was also used for hybrid Vlasovfluid models in [44]. Further details can be found in [12], where similar Lie group structures were shown to arise in polymer dynamics.

At this point, general geometric mechanics arguments ensure that the EulerPoincaré variational principle $\delta \int_{t_{0}}^{t_{1}} l(\boldsymbol{U}, \boldsymbol{V}, \mathbf{X}, \rho, \mathbf{A}, f) \mathrm{d} t=0$ produces the following equations of motion:

$$
\begin{aligned}
& \frac{\partial}{\partial t} \frac{\delta l}{\delta \boldsymbol{U}}+£_{\boldsymbol{U}} \frac{\delta l}{\delta \boldsymbol{U}}=-£_{\boldsymbol{V}} \frac{\delta l}{\delta \boldsymbol{V}}+\frac{\delta l}{\delta \mathbf{X}} \star \mathbf{X}+\left(\frac{\delta l}{\delta \rho}, \frac{\delta l}{\delta \mathbf{A}}, \frac{\delta l}{\delta f}\right) \diamond_{1}(\rho, \mathbf{A}, f), \\
& \frac{\partial \rho}{\partial t}+£_{\boldsymbol{U}} \rho=0, \quad \frac{\partial \mathbf{A}}{\partial t}+£_{\boldsymbol{U}} \mathbf{A}=0 \\
& \frac{\partial}{\partial t} \frac{\delta l}{\delta \boldsymbol{V}}+£_{\boldsymbol{V}+\boldsymbol{U}} \frac{\delta l}{\delta \boldsymbol{V}}=\frac{\delta l}{\delta \mathbf{X}} \star \mathbf{X}+\frac{\delta l}{\delta f} \diamond_{1} f, \\
& \frac{\partial}{\partial t} \frac{\delta l}{\delta \mathbf{X}}+£_{\mathbf{X}+\mathbf{X}_{\boldsymbol{V}+U}} \frac{\delta l}{\delta \mathbf{X}}=f \nabla_{(\mathbf{x}, \mathbf{v})} \frac{\delta l}{\delta f} \\
& \frac{\partial f}{\partial t}+£_{\mathbf{X}+\mathbf{X}_{\boldsymbol{V}+\boldsymbol{U}}} f=0 .
\end{aligned}
$$

In order to see how equations (5.6)-(5.10) recover the second pressure coupling scheme (5.1)-(5.3), it suffices to substitute the Lagrangian (5.4). After computing

$$
\begin{gathered}
\frac{\delta l}{\delta \mathbf{u}}=m_{h} f\left(2 \mathbf{u}-\mathbf{v}+a_{h} \mathbf{A}\right), \quad \frac{\delta l}{\delta \boldsymbol{\alpha}}=0, \\
\frac{\delta l}{\delta f}=\frac{m_{h}}{2}|\mathbf{u}|^{2}+\frac{m_{h}}{2}|\mathbf{u}-\mathbf{v}|^{2}-\frac{m_{h}}{2}|\boldsymbol{V}|^{2}+q_{h}(\mathbf{u}+\boldsymbol{V}) \cdot \mathbf{A},
\end{gathered}
$$

the second component of equation (4.10) yields $\mathbf{u}=\mathbf{v}$ so that $\mathbf{X}=(\mathbf{v}, \boldsymbol{\alpha}(\mathbf{x}, \mathbf{v}))$, similarly to the results in the previous section. Moreover, the first component of (4.10) reads as

$$
\partial_{t} \boldsymbol{p}+\left((\mathbf{v}+\boldsymbol{V}+\boldsymbol{U}) \cdot \partial_{\mathbf{x}}\right) \boldsymbol{p}+\left(\mathbf{a} \cdot \partial_{\mathbf{v}}\right) \boldsymbol{p}+\partial_{\mathbf{x}}(\mathbf{v}+\boldsymbol{V}+\boldsymbol{U}) \cdot \boldsymbol{p}=\partial_{\mathbf{x}}\left(q_{h}(\mathbf{v}+\boldsymbol{V}) \cdot \mathbf{A}-\frac{m_{h}}{2}|\boldsymbol{V}|^{2}\right),
$$

where we have denoted by $\mathbf{a}=\boldsymbol{\alpha}+\left(\mathbf{X}_{\boldsymbol{V}+\boldsymbol{U}}\right)_{2}=\boldsymbol{\alpha}+(\mathbf{v} \cdot \nabla)(\boldsymbol{V}+\boldsymbol{U})$ the total acceleration of the hot particles. After using standard vector identities, this equation yields the expression for the total force on the hot component:

$$
m_{h} \mathbf{a}=q_{h}(\mathbf{v}+\boldsymbol{V}) \times \mathbf{B}-m_{h} \nabla \boldsymbol{U} \cdot \mathbf{v}-m_{h} \nabla \boldsymbol{V} \cdot(\mathbf{v}+\boldsymbol{V}) .
$$


Then, the total vector field $\mathbf{X}+\mathbf{X}_{\boldsymbol{V}+\boldsymbol{U}}=(\mathbf{v}+\boldsymbol{V}+\boldsymbol{U}$, a) is divergence-free and the Vlasov equation (4.10) becomes

$$
\frac{\partial f}{\partial t}+(\mathbf{v}+\boldsymbol{V}+\boldsymbol{U}) \cdot \frac{\partial f}{\partial \mathbf{x}}+\left(a_{h}(\mathbf{v}+\boldsymbol{V}) \times \mathbf{B}-\nabla \boldsymbol{U} \cdot \mathbf{v}-\nabla \boldsymbol{V} \cdot(\mathbf{v}+\boldsymbol{V})\right) \cdot \frac{\partial f}{\partial \mathbf{v}}=0 .
$$

At this point, we observe that the constraint $\boldsymbol{V}=-\mathbf{K} / n$ is preserved by the dynamics. This is a direct consequence of the following

Proposition 5.1. With the notation of Lemma 4.5, the Euler-Poincaré equations (5.6)-(5.10) yield

$$
\left(\frac{\partial}{\partial t}+£_{\boldsymbol{V}+\boldsymbol{U}}\right)\left(\frac{\delta l}{\delta \boldsymbol{V}}-i^{*}\left(\frac{\delta l}{\delta \mathbf{X}}\right)\right)=0 .
$$

The proof proceeds analogously to that of Proposition 4.4 (see also Lemma 4.5). Upon considering the Lagrangian (5.4), the Euler-Poincaré equations (5.6)-(5.10) preserve the constraint

$$
-\boldsymbol{V} \int f \mathrm{~d}^{3} \mathbf{v}=\int \mathbf{v} f \mathrm{~d}^{3} \mathbf{v}
$$

which allows one to recover the Vlasov equation (5.2) of the second pressure coupling scheme in [44]. Analogously, one can show that equation (5.6) recovers the hybrid equation of motion of the same pressure coupling scheme, that is (5.1). In order to show this, it suffices to verify that

$$
-£_{\boldsymbol{V}} \frac{\delta l}{\delta \boldsymbol{V}}+\frac{\delta l}{\delta f} \diamond_{1} f=-m_{h} \nabla \cdot \int\left(\mathbf{v}-\frac{\mathbf{K}}{n}\right)\left(\mathbf{v}-\frac{\mathbf{K}}{n}\right) f \mathrm{~d}^{3} \mathbf{v}=:-m_{h} \nabla \cdot \overline{\mathbb{P}} .
$$

This formula requires a lengthy but straightforward calculation that uses $\boldsymbol{V}=-n^{-1} \mathbf{K}$ and the well known relation

$$
\nabla \cdot \int \mathbf{v v} f \mathrm{~d}^{3} \mathbf{v}=\nabla \cdot\left(n^{-1} \mathbf{K K}+\int\left(\mathbf{v}-n^{-1} \mathbf{K}\right)\left(\mathbf{v}-n^{-1} \mathbf{K}\right) f \mathrm{~d}^{3} \mathbf{v}\right)
$$

between the absolute and relative pressure tensors. Thus, in conclusion, we have proven the following

THEOREM 5.2. The hybrid pressure-coupling MHD scheme (5.1)-(5.3) arises from the Euler-Poincaré variational principle

$$
\delta \int_{t_{0}}^{t_{1}} l(\boldsymbol{U}, \boldsymbol{V}, \mathbf{X}, \rho, \mathbf{A}, f) \mathrm{d} t=0,
$$

with the reduced Lagrangian

$$
l: \mathfrak{X}_{1}\left(\mathbb{R}^{3}\right)\left(S\left(\mathfrak{X}_{2}\left(\mathbb{R}^{3}\right) \subseteq \mathfrak{X}\left(\mathbb{R}^{6}\right)\right) \times C^{\infty}\left(\mathbb{R}^{3}\right)^{*} \times \Omega^{1}\left(\mathbb{R}^{3}\right) \times C^{\infty}\left(\mathbb{R}^{6}\right)^{*} \rightarrow \mathbb{R}\right.
$$

as in (5.4) and variations

$$
\begin{aligned}
& \delta(\boldsymbol{U}, \boldsymbol{V}, \mathbf{X})=\partial_{t}(\mathbf{W}, \mathbf{P}, \mathbf{Z})-\left(£_{\boldsymbol{U}} \mathbf{W}, £_{\mathbf{W}} \boldsymbol{V}-£_{\boldsymbol{U}} \mathbf{P}+£_{\boldsymbol{V}} \mathbf{P}, £_{\mathbf{X}_{\mathbf{P}+\mathbf{W}}} \mathbf{X}-£_{\mathbf{X}_{\boldsymbol{V}+U}} \mathbf{Z}+£_{\mathbf{X}} \mathbf{Z}\right),
\end{aligned}
$$

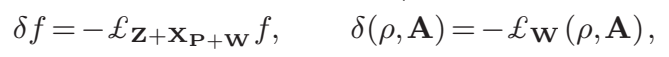

in which the vector fields $\mathbf{P}, \mathbf{W} \in \mathfrak{X}\left(\mathbb{R}^{3}\right)$ and $\mathbf{Z} \in \mathfrak{X}\left(\mathbb{R}^{6}\right)$ vanish at the endpoints. This variational principle is equivalent to the Euler-Poincaré equations (5.6)-(5.10) which hold for an arbitrary hybrid Lagrangian. 
5.3. Discussion. It is relevant to notice that equations (5.6) and (5.9) yield the following relation:

$$
\begin{aligned}
\left(\frac{\partial}{\partial t}+£_{\boldsymbol{U}}\right)\left(\frac{\delta l}{\delta \boldsymbol{U}}-i^{*}\left(\frac{\delta l}{\delta \mathbf{X}}\right)\right)=-£_{\boldsymbol{V}}\left(\frac{\delta l}{\delta \boldsymbol{V}}\right. & \left.-i^{*}\left(\frac{\delta l}{\delta \mathbf{X}}\right)\right)+\rho \nabla \frac{\delta l}{\delta \rho} \\
& -\frac{\delta l}{\delta \mathbf{A}} \times \nabla \times \mathbf{A}+\left(\nabla \cdot \frac{\delta l}{\delta \mathbf{A}}\right) \mathbf{A},
\end{aligned}
$$

where one has used Lemma 4.5. Upon inserting the Lagrangian (5.4), the Lie derivatives in the right hand side cancel due to the constraint $\boldsymbol{V}=-\mathbf{K} / n$.

Kelvin circulation laws. The Kelvin-Noether conservation laws also hold for equations (5.1)-(5.3). Indeed, upon repeating the same steps as in the proof of Corollary 4.6 , one finds the circulation laws

$$
\begin{aligned}
& \frac{\mathrm{d}}{\mathrm{d} t} \oint_{\gamma_{t}(\boldsymbol{U})} \boldsymbol{U} \cdot \mathrm{d} \mathbf{x}=-\oint_{\gamma_{t}(\boldsymbol{U})} \frac{1}{\rho}\left(\frac{1}{\mu_{0}} \mathbf{B} \times \nabla \times \mathbf{B}+m_{h} \nabla \cdot \overline{\mathbb{P}}\right) \cdot \mathrm{d} \mathbf{x}, \\
& \frac{\mathrm{d}}{\mathrm{d} t} \oint_{\gamma_{t}(\boldsymbol{U})}\left(\boldsymbol{U}-\frac{1}{\rho} \int f \boldsymbol{p} \mathrm{d}^{3} \mathbf{v}\right) \cdot \mathrm{d} \mathbf{x}=-\mu_{0}^{-1} \oint_{\gamma_{t}(\boldsymbol{U})} \frac{1}{\rho} \mathbf{B} \times \nabla \times \mathbf{B} \cdot \mathrm{d} \mathbf{x},
\end{aligned}
$$

where the second is a direct consequence of equation (5.13). These results coincide with those found in [44] within the Lie-Poisson Hamiltonian setting. Taking the difference of the above two relations yields

$$
\frac{\mathrm{d}}{\mathrm{d} t} \oint_{\gamma_{t}(\boldsymbol{U})}\left(\frac{1}{\rho} \int f \boldsymbol{p} \mathrm{d}^{3} \mathbf{v}\right) \cdot \mathrm{d} \mathbf{x}=m_{h} \frac{\mathrm{d}}{\mathrm{d} t} \oint_{\gamma_{t}(\boldsymbol{U})} \frac{\mathbf{K}}{\rho} \cdot \mathrm{d} \mathbf{x}=-m_{h} \oint_{\gamma_{t}(\boldsymbol{U})} \frac{1}{\rho}(\nabla \cdot \overline{\mathbb{P}}) \cdot \mathrm{d} \mathbf{x},
$$

where we have used the zero-th moment equation $\partial_{t} n+\nabla \cdot(n \boldsymbol{U})=0$ associated to (5.11). Indeed, together with equation (5.7), this yields the following conserved circulation:

$$
\frac{\mathrm{d}}{\mathrm{d} t} \oint_{\gamma_{t}(\boldsymbol{U})}\left(1+\frac{n}{\rho}\right) \mathbf{A} \cdot \mathrm{d} \mathbf{x}=0
$$

Moreover, the above circulation laws are accompanied by the following Poincaré invariant relation:

$$
\frac{\mathrm{d}}{\mathrm{d} t} \oint_{\zeta_{t}\left(\mathbf{X}+\mathbf{X}_{U-\mathrm{K} / n}\right)} \boldsymbol{p} \cdot \mathrm{d} \mathbf{x}=0
$$

where the curve $\zeta_{t}$ moves along the total phase-space vector field $\mathbf{X}+\mathbf{X}_{U-\mathbf{K} / n}$.

Ertel's relation. Proceeding as in Section 3.3, taking the curl of the incompressible version of equation (5.13) for the Lagrangian (4.13), or equivalently applying the Stokes theorem in the Kelvin-Noether relation (5.15) produces an Ertel relation of the form

$$
D_{t}(\overline{\boldsymbol{\omega}} \cdot \nabla \alpha)-(\overline{\boldsymbol{\omega}} \cdot \nabla) D_{t} \alpha=-\mu_{0}^{-1} \nabla \alpha \cdot \nabla \times(\mathbf{B} \times(\nabla \times \mathbf{B})),
$$

where $\alpha$ is an arbitrary scalar function and, upon keeping the mass density $\rho$ (equal to one for incompressible flows),

$$
\overline{\boldsymbol{\omega}}=\nabla \times\left(\boldsymbol{U}-m_{h} \frac{\mathbf{K}}{\rho}\right) .
$$


The Ertel relation above, written in terms of $\overline{\boldsymbol{\omega}}$, has the same form as the corresponding relation in [15], except that the vorticity $\bar{\omega}$ here involves a velocity shift due to the mean specific momentum carried by the particles.

Cross helicities. Upon denoting $\mathcal{W}=\boldsymbol{U}-\rho^{-1} \int f \boldsymbol{p} \mathrm{d}^{3} \mathbf{v}$, it easy to see that the two cross helicities

$$
\Lambda_{1}=\int \boldsymbol{U} \cdot \mathbf{B} \mathrm{d}^{3} \mathbf{x} \quad \text { and } \quad \Lambda_{3}=\int \mathcal{W} \cdot \mathbf{B} \mathrm{d}^{3} \mathbf{x}
$$

possess the following dynamics:

$$
\frac{\mathrm{d} \Lambda_{1}}{\mathrm{~d} t}=-m_{h} \int \rho^{-1}(\nabla \cdot \overline{\mathbb{P}}) \cdot \mathbf{B} \mathrm{d}^{3} \mathbf{x}, \quad \frac{\mathrm{d} \Lambda_{3}}{\mathrm{~d} t}=0,
$$

so that $\Lambda_{3}$ is now conserved by the hybrid dynamics of equations (5.1)-(5.3). Also, notice the conservation of the modified magnetic helicity

$$
\mathcal{H}=\int \frac{n}{\rho} \mathbf{A} \cdot \mathbf{B} \mathrm{d}^{3} \mathbf{x}
$$

which allows to write the cross helicity invariant $\Lambda_{3}$ as

$$
\Lambda_{3}=\int\left(\boldsymbol{U}-m_{h} \frac{\mathbf{K}}{\rho}\right) \cdot \mathbf{B} \mathrm{d}^{3} \mathbf{x} .
$$

The conservation law for $\mathcal{H}$ provides an interesting opportunity to study the stability properties of this hybrid scheme. In particular, $\mathcal{H}$ does not vanish for static equilibria. This means the energy-Casimir method may be applied for hybrid fluid equilibria that are analogous to the Chandrasekhar flows of inviscid MHD [22].

\section{Summary and conclusions}

This paper has derived three different hybrid Vlasov-fluid plasma models by using the Euler-Poincaré approach first developed for the Maxwell-Vlasov plasma [7]. After presenting the Euler-Poincaré approach for the Vlasov-multifluid plasma system, the discussion focused on three different schemes for deriving hybrid Vlasov-fluid MHD models. These comprised the current-coupling scheme and two pressure-coupling schemes. The first hybrid model was written on the direct product of two different diffeomorphism groups, as explained in Remark 3.2. The second one involved the more sophisticated construction of the semidirect-product diffeomorphism group discussed in Remark 4.3. Finally, a compound semidirect-product structure arose for the third hybrid model; see equation (5.5). In all three theories, Kelvin circulation theorems were presented for both the fluid motion and the hot particle dynamics on phase space, and the invariant cross-helicities were identified. A Legendre transform in each case would recover the Lie-Poisson results found [44]. Shifting to the drift-kinetic (or even gyrokinetic) approximation would require another Lagrangian, which may also be derived systematically from the Lagrangian for Vlasov-MHD. Summaries of the properties found here for the three different hybrid Vlasov-fluid plasma models are given below. 


\section{Current-coupling MHD scheme}

- Equations of motion (3.6)-(3.9):

$$
\begin{aligned}
& \rho \frac{\partial \boldsymbol{U}}{\partial t}+\rho(\boldsymbol{U} \cdot \nabla) \boldsymbol{U}=\left(q_{h} n \boldsymbol{U}-q_{h} \mathbf{K}+\frac{1}{\mu_{0}} \nabla \times \mathbf{B}\right) \times \mathbf{B}-\nabla \mathbf{p} \\
& \frac{\partial f}{\partial t}+\mathbf{v} \cdot \frac{\partial f}{\partial \mathbf{x}}+a_{h}(\mathbf{v}-\boldsymbol{U}) \times \mathbf{B} \cdot \frac{\partial f}{\partial \mathbf{v}}=0 \\
& \frac{\partial \rho}{\partial t}+\nabla \cdot(\rho \boldsymbol{U})=0, \quad \frac{\partial \mathbf{B}}{\partial t}=\nabla \times(\boldsymbol{U} \times \mathbf{B})
\end{aligned}
$$

- Kelvin circulation laws derived from (3.16)-(3.18):

$$
\begin{aligned}
& \frac{\mathrm{d}}{\mathrm{d} t} \oint_{\gamma_{t}} \boldsymbol{U} \cdot \mathrm{d} \mathbf{x}=\oint_{\gamma_{t}} \frac{1}{\rho}\left(q_{h} n \boldsymbol{U}-q_{h} \mathbf{K}+\frac{1}{\mu_{0}} \nabla \times \mathbf{B}\right) \times \mathbf{B} \cdot \mathrm{d} \mathbf{x}, \\
& \frac{\mathrm{d}}{\mathrm{d} t} \oint_{\gamma_{t}}\left(1+\frac{n}{\rho}\right) \mathbf{A} \cdot \mathrm{d} \mathbf{x}=\oint_{\gamma_{t}} \frac{1}{\rho}(\nabla \cdot(n \boldsymbol{U}-\mathbf{K})) \mathbf{A} \cdot \mathrm{d} \mathbf{x} .
\end{aligned}
$$

where $\gamma_{t}$ is any closed loop that moves with the fluid velocity $\boldsymbol{U}$;

- Magnetic and cross helicity invariants:

$$
\mathcal{H}=\int \mathbf{A} \cdot \mathbf{B} \mathrm{d}^{3} \mathbf{x}, \quad \Lambda=\int \boldsymbol{U} \cdot \mathbf{B} \mathrm{d}^{3} \mathbf{x}
$$

- Approximation: same as ideal MHD; this yields the Lagrangian (3.10).

\section{Pressure-coupling MHD scheme - first variant}

- Equations of motion (4.3)-(4.5):

$$
\begin{aligned}
& \rho \frac{\partial \boldsymbol{U}}{\partial t}+\rho(\boldsymbol{U} \cdot \nabla) \boldsymbol{U}=-\nabla \mathbf{p}-m_{h} \nabla \cdot \mathbb{P}-\frac{1}{\mu_{0}} \mathbf{B} \times \nabla \times \mathbf{B}, \\
& \frac{\partial f}{\partial t}+(\boldsymbol{U}+\mathbf{v}) \cdot \frac{\partial f}{\partial \mathbf{x}}-\frac{\partial f}{\partial \mathbf{v}} \cdot \nabla \boldsymbol{U} \cdot \mathbf{v}+a_{h} \mathbf{v} \times \mathbf{B} \cdot \frac{\partial f}{\partial \mathbf{v}}=0, \\
& \frac{\partial \rho}{\partial t}+\nabla \cdot(\rho \boldsymbol{U})=0, \quad \frac{\partial \mathbf{B}}{\partial t}=\nabla \times(\boldsymbol{U} \times \mathbf{B})
\end{aligned}
$$

- Kelvin circulation laws (4.16)-(4.17):

$$
\begin{aligned}
& \frac{\mathrm{d}}{\mathrm{d} t} \oint_{\gamma_{t}} \boldsymbol{U} \cdot \mathrm{d} \mathbf{x}=-\oint_{\gamma_{t}} \frac{1}{\rho}\left(\frac{1}{\mu_{0}} \mathbf{B} \times \nabla \times \mathbf{B}+m_{h} \nabla \cdot \mathbb{P}\right) \cdot \mathrm{d} \mathbf{x}, \\
& \frac{\mathrm{d}}{\mathrm{d} t} \oint_{\gamma_{t}} \frac{\mathbf{K}}{\rho} \cdot \mathrm{d} \mathbf{x}=\oint_{\gamma_{t}} \frac{1}{\rho}\left(a_{h} \mathbf{K} \times \mathbf{B}-\nabla \cdot \mathbb{P}\right) \cdot \mathrm{d} \mathbf{x}, \\
& \frac{\mathrm{d}}{\mathrm{d} t} \oint_{\gamma_{t}}\left(1+\frac{n}{\rho}\right) \mathbf{A} \cdot \mathrm{d} \mathbf{x}=-\oint_{\gamma_{t}} \frac{1}{\rho}(\nabla \cdot \mathbf{K}) \mathbf{A} \cdot \mathrm{d} \mathbf{x} ;
\end{aligned}
$$

- Magnetic and cross helicity invariants:

$$
\mathcal{H}=\int \mathbf{A} \cdot \mathbf{B} \mathrm{d}^{3} \mathbf{x}, \quad \Lambda=\int\left(\boldsymbol{U}-m_{h} \frac{\mathbf{K}}{\rho}\right) \cdot \mathbf{B} \mathrm{d}^{3} \mathbf{x} ;
$$


- Approximation: neglects minimal coupling term $\int n \boldsymbol{U} \cdot \mathbf{A d} \mathrm{d}^{3} \mathbf{x}$ in the Lagrangian (3.10).

\section{Pressure-coupling MHD scheme - second variant}

- Equations of motion (5.1)-(5.3):

$$
\begin{aligned}
& \rho \frac{\partial \boldsymbol{U}}{\partial t}+\rho(\boldsymbol{U} \cdot \nabla) \boldsymbol{U}=-\nabla \mathbf{p}-m_{h} \nabla \cdot \overline{\mathbb{P}}-\frac{1}{\mu_{0}} \mathbf{B} \times \nabla \times \mathbf{B}, \\
& \frac{\partial f}{\partial t}+\left(\mathbf{v}+\boldsymbol{U}-\frac{\mathbf{K}}{n}\right) \cdot \frac{\partial f}{\partial \mathbf{x}} \\
& \quad+\left(a_{h}\left(\mathbf{v}-\frac{\mathbf{K}}{n}\right) \times \mathbf{B}-\nabla \boldsymbol{U} \cdot \mathbf{v}+\left(\nabla \frac{\mathbf{K}}{n}\right) \cdot\left(\mathbf{v}-\frac{\mathbf{K}}{n}\right)\right) \cdot \frac{\partial f}{\partial \mathbf{v}}=0, \\
& \frac{\partial \rho}{\partial t}+\nabla \cdot(\rho \boldsymbol{U})=0, \quad \frac{\partial \mathbf{B}}{\partial t}=\nabla \times(\boldsymbol{U} \times \mathbf{B}) ;
\end{aligned}
$$

- Kelvin circulation laws (5.14)-(5.15):

$$
\begin{aligned}
& \frac{\mathrm{d}}{\mathrm{d} t} \oint_{\gamma_{t}} \boldsymbol{U} \cdot \mathrm{d} \mathbf{x}=-\oint_{\gamma_{t}} \frac{1}{\rho}\left(\frac{1}{\mu_{0}} \mathbf{B} \times \nabla \times \mathbf{B}+m_{h} \nabla \cdot \overline{\mathbb{P}}\right) \cdot \mathrm{d} \mathbf{x} \\
& \frac{\mathrm{d}}{\mathrm{d} t} \oint_{\gamma_{t}} \frac{\mathbf{K}}{\rho} \cdot \mathrm{d} \mathbf{x}=-\oint_{\gamma_{t}} \frac{1}{\rho}(\nabla \cdot \overline{\mathbb{P}}) \cdot \mathrm{d} \mathbf{x}, \quad \frac{\mathrm{d}}{\mathrm{d} t} \oint_{\gamma_{t}}\left(1+\frac{n}{\rho}\right) \mathbf{A} \cdot \mathrm{d} \mathbf{x}=0
\end{aligned}
$$

- Magnetic and cross helicity invariants:

$$
\mathcal{H}=\int\left(1+\frac{n}{\rho}\right) \mathbf{A} \cdot \mathbf{B} \mathrm{d}^{3} \mathbf{x}, \quad \Lambda=\int\left(\boldsymbol{U}-m_{h} \frac{\mathbf{K}}{\rho}\right) \cdot \mathbf{B} \mathrm{d}^{3} \mathbf{x}
$$

- Approximation: neglects $\int n \boldsymbol{U} \cdot \mathbf{A d} \mathrm{d}^{3} \mathbf{x}$ as well as mean flow terms in the Lagrangian (3.10).

The Euler-Poincaré approach provided the means of comparing the geometrical properties of these three hybrid Vlasov-fluid plasma schemes in the same framework. This framework allowed the identification and comparison of the geometric relationships within each scheme that were shared by the others. We expect that this framework will be useful in other modeling contexts. For example, one may imagine using the Euler-Poincaré framework (i) in the comparison and selection of Vlasov-fluid hybrid models, (ii) in the validation of previous derivations, (iii) in making choices among the schemes in various physical regimes, and (iv) as a basis for performing other derivations obtained by modeling in the Lagrangian. From the physical viewpoint the roles of heat exchange and other advected quantities should also lead to interesting effects in future investigations. For example, the introduction of another advected quantity would produce an explicit Ertel theorem for the evolution of potential vorticity. Of course, Ertel's theorem is an immediate result of the Euler-Poincaré theory for any hybrid fluid-Vlasov model with advected quantities. However, it was discussed here only in the simple case of incompressible hybrid fluid flows.

In another direction for future research in the context of potential vorticity, one may use Ertel's theorem to investigate the evolution of the gradient of the potential vorticity, as studied recently in geophysical fluid dynamics in terms of the vector 
$\mathcal{B}=\nabla Q(q) \times \nabla \alpha[13]$. Here $Q$ is an arbitrary function and $q=\overline{\boldsymbol{\omega}} \cdot \nabla \alpha$ is the potential vorticity arising in a given fluid model. Upon considering an advected function $\alpha$ such that $\partial_{t} \alpha+\boldsymbol{U} \cdot \nabla \alpha=0$, Ertel's theorem was shown in [13] to produce the dynamics of the vector $\mathcal{B}$ in the form

$$
\frac{\partial \mathcal{B}}{\partial t}-\nabla \times(\mathcal{U} \times \mathcal{B})=\nabla \times \boldsymbol{\Phi}
$$

where $\mathcal{U}=\boldsymbol{U}-q^{-1}(\alpha \nabla \times \boldsymbol{\Psi})$ and $\nabla \times \boldsymbol{\Phi}=\nabla \alpha \times \nabla\left(q Q^{\prime} \nabla \cdot \mathcal{U}\right)$. Evidently, the divergenceless vector $\mathcal{D}=\nabla \times \boldsymbol{\Phi}$ breaks the frozen-in condition of the vector $\mathcal{B}$ and it thus affects the stretching properties that are governed by the left-hand side of the equation for the vector $\mathcal{B}$; see [13]. The identification of the vorticity $\overline{\boldsymbol{\omega}}$ and the force $\boldsymbol{\Psi}$ in each of the hybrid models discussed here would provide interesting opportunities to study the dynamics of the gradients of the potential vorticity. This would perhaps lead to the production of fronts and other fine structures at high wave numbers that develop by stretching of the $\mathcal{B}$-vector.

Acknowledgment. We are grateful to C. David Levermore for many inspiring discussions on these and related topics over the years. This paper is dedicated to him on the occasion of his sixtieth birthday. Happy birthday, Dave! DDH was partially supported by the Royal Society of London Wolfson Scheme and the European Research Council Advanced Investigator Grant.

\section{Appendix A. Proof of Lemma 4.5.}

Proof. The map $i^{*}$ is easily seen to be a momentum map arising from the dual of the Lie algebra inclusion $i: \boldsymbol{U} \mapsto \mathbf{X}_{\boldsymbol{U}}$. Upon denoting $\boldsymbol{\Xi}=\delta l / \delta \mathbf{X}$, the momentum map property

$$
\left\{F,\left\langle i^{*}(\boldsymbol{\Xi}), \boldsymbol{U}\right\rangle\right\}_{\mathfrak{X}^{*}\left(\mathbb{R}^{6}\right)}=\boldsymbol{U}_{\mathcal{F}\left(\mathfrak{X}^{*}\left(\mathbb{R}^{6}\right)\right)}[F]
$$

can be verified explicitly. Here, $\boldsymbol{U}_{\mathcal{F}\left(\mathfrak{X}^{*}\left(\mathbb{R}^{6}\right)\right)}[F]$ denotes evaluation on the functional $F(\boldsymbol{\Xi})$ of the infinitesimal generator $\boldsymbol{U}_{\mathcal{F}\left(\mathfrak{X}^{*}\left(\mathbb{R}^{6}\right)\right)}$ of the Diff $\left(\mathbb{R}^{3}\right)$-action on the space of functionals $\mathcal{F}\left(\mathfrak{X}^{*}\left(\mathbb{R}^{6}\right)\right.$ ) on the one-form densities in $\mathfrak{X}^{*}\left(\mathbb{R}^{6}\right)$. Upon using the right Lie-Poisson bracket on $\mathfrak{X}^{*}\left(\mathbb{R}^{6}\right)$, one computes

$$
\begin{aligned}
\left\{F,\left\langle i^{*}(\boldsymbol{\Xi}), \boldsymbol{U}\right\rangle\right\}_{\mathfrak{X}^{*}\left(\mathbb{R}^{6}\right)} & =\left\langle\boldsymbol{\Xi},\left[\frac{\delta F}{\delta \boldsymbol{\Xi}}, \frac{\delta}{\delta \boldsymbol{\Xi}}\left\langle i^{*}(\boldsymbol{\Xi}), \boldsymbol{U}\right\rangle\right]\right\rangle_{\mathfrak{X}^{*}\left(\mathbb{R}^{6}\right)} \\
& =\left\langle\boldsymbol{\Xi},\left[\frac{\delta F}{\delta \boldsymbol{\Xi}}, \frac{\delta}{\delta \boldsymbol{\Xi}}\langle\boldsymbol{\Xi}, i(\boldsymbol{U})\rangle\right]\right\rangle_{\mathfrak{X}^{*}\left(\mathbb{R}^{6}\right)} \\
& =\left\langle\boldsymbol{\Xi}, £_{i(\boldsymbol{U})} \frac{\delta F}{\delta \boldsymbol{\Xi}}\right\rangle_{\mathfrak{X}^{*}\left(\mathbb{R}^{6}\right)} \\
& =-\left\langle £_{\mathbf{X}_{U}} \boldsymbol{\Xi}, \frac{\delta F}{\delta \mathbf{\Xi}}\right\rangle_{\mathfrak{X}^{*}\left(\mathbb{R}^{6}\right)} \\
& =\boldsymbol{U}_{\mathcal{F}\left(\mathfrak{X}^{*}\left(\mathbb{R}^{6}\right)\right)}[F] .
\end{aligned}
$$

where $[\cdot, \cdot]$ denotes minus the Jacobi-Lie bracket on $\mathfrak{X}\left(\mathbb{R}^{6}\right)$. 
The rest of the proof proceeds in two steps. First,

$$
\begin{aligned}
& \int\left(£_{\mathbf{X}_{U}} \frac{\delta l}{\delta \mathbf{X}}\right)_{1} \mathrm{~d}^{3} \mathbf{v} \\
= & \int\left(\boldsymbol{U} \cdot \partial_{\mathbf{x}}+(\mathbf{v} \cdot \nabla) \boldsymbol{U} \cdot \partial_{\mathbf{v}}\right) \frac{\delta l}{\delta \mathbf{u}} \mathrm{d}^{3} \mathbf{v} \\
& +\int\left(\nabla \boldsymbol{U} \cdot \frac{\delta l}{\delta \mathbf{u}}+\partial_{\mathbf{x}}((\mathbf{v} \cdot \nabla) \boldsymbol{U}) \cdot \frac{\delta l}{\delta \boldsymbol{\alpha}}+\left(\nabla \cdot \boldsymbol{U}+\partial_{\mathbf{v}} \cdot((\mathbf{v} \cdot \nabla) \boldsymbol{U})\right) \frac{\delta l}{\delta \mathbf{u}}\right) \mathrm{d}^{3} \mathbf{v} \\
= & \int\left(\boldsymbol{U} \cdot \partial_{\mathbf{x}}\right) \frac{\delta l}{\delta \mathbf{u}} \mathrm{d}^{3} \mathbf{v}+\int\left((\mathbf{v} \cdot \nabla) \boldsymbol{U} \cdot \partial_{\mathbf{v}}\right) \frac{\delta l}{\delta \mathbf{u}} \mathrm{d}^{3} \mathbf{v}+\int((\mathbf{v} \cdot \nabla) \nabla \boldsymbol{U}) \cdot \frac{\delta l}{\delta \boldsymbol{\alpha}} \mathrm{d}^{3} \mathbf{v} \\
& +\int\left(\nabla \boldsymbol{U} \cdot \frac{\delta l}{\delta \mathbf{u}}+(\nabla \cdot \boldsymbol{U}) \frac{\delta l}{\delta \mathbf{u}}\right) \mathrm{d}^{3} \mathbf{v}-\int\left(\left((\mathbf{v} \cdot \nabla) \boldsymbol{U} \cdot \partial_{\mathbf{v}}\right) \frac{\delta l}{\delta \mathbf{u}}\right) \mathrm{d}^{3} \mathbf{v} \\
= & £_{\boldsymbol{U}} \int \frac{\delta l}{\delta \mathbf{u}} \mathrm{d}^{3} \mathbf{v}+\int((\mathbf{v} \cdot \nabla) \nabla \boldsymbol{U}) \cdot \frac{\delta l}{\delta \boldsymbol{\alpha}} \mathrm{d}^{3} \mathbf{v} .
\end{aligned}
$$

One also has

$$
\begin{aligned}
\int\left(\mathbf{v} \cdot \partial_{\mathbf{x}}\right)\left(£_{\mathbf{X}_{U}} \frac{\delta l}{\delta \mathbf{X}}\right)_{2} \mathrm{~d}^{3} \mathbf{v}= & \int\left(\mathbf{v} \cdot \partial_{\mathbf{x}}\right)\left(\left(\boldsymbol{U} \cdot \partial_{\mathbf{x}}+(\mathbf{v} \cdot \nabla) \boldsymbol{U} \cdot \partial_{\mathbf{v}}\right) \frac{\delta l}{\delta \boldsymbol{\alpha}}\right) \mathrm{d}^{3} \mathbf{v} \\
& +\int\left(\mathbf{v} \cdot \partial_{\mathbf{x}}\right)\left(\partial_{\mathbf{v}}((\mathbf{v} \cdot \nabla) \boldsymbol{U}) \cdot \frac{\delta l}{\delta \boldsymbol{\alpha}}\right) \mathrm{d}^{3} \mathbf{v} \\
& +\int\left(\mathbf{v} \cdot \partial_{\mathbf{x}}\right)\left(\left(\nabla \cdot \boldsymbol{U}+\partial_{\mathbf{v}} \cdot((\mathbf{v} \cdot \nabla) \boldsymbol{U})\right) \frac{\delta l}{\delta \boldsymbol{\alpha}}\right) \mathrm{d}^{3} \mathbf{v} .
\end{aligned}
$$

Then, for each term, one computes

$$
\begin{aligned}
& \int\left(\mathbf{v} \cdot \partial_{\mathbf{x}}\right)\left(\left(\boldsymbol{U} \cdot \partial_{\mathbf{x}}\right) \frac{\delta l}{\delta \boldsymbol{\alpha}}\right) \mathrm{d}^{3} \mathbf{v}=\boldsymbol{U} \cdot \nabla \int\left(\mathbf{v} \cdot \partial_{\mathbf{x}}\right) \frac{\delta l}{\delta \boldsymbol{\alpha}} \mathrm{d}^{3} \mathbf{v}+\int \operatorname{Tr}\left((\nabla \boldsymbol{U})\left(\mathbf{v} \partial_{\mathbf{x}}\right)\right) \frac{\delta l}{\delta \boldsymbol{\alpha}} \mathrm{d}^{3} \mathbf{v}, \\
& \int\left(\mathbf{v} \cdot \partial_{\mathbf{x}}\right)\left(\left(\partial_{\mathbf{v}}((\mathbf{v} \cdot \nabla) \boldsymbol{U})\right) \frac{\delta l}{\delta \boldsymbol{\alpha}}\right) \mathrm{d}^{3} \mathbf{v}=\nabla \boldsymbol{U} \cdot \int\left(\mathbf{v} \cdot \partial_{\mathbf{x}}\right) \frac{\delta l}{\delta \boldsymbol{\alpha}} \mathrm{d}^{3} \mathbf{v}+\int((\mathbf{v} \cdot \nabla) \nabla \boldsymbol{U}) \cdot \frac{\delta l}{\delta \boldsymbol{\alpha}} \mathrm{d}^{3} \mathbf{v}, \\
& \int\left(\mathbf{v} \cdot \partial_{\mathbf{x}}\right)\left((\nabla \cdot \boldsymbol{U}) \frac{\delta l}{\delta \boldsymbol{\alpha}}\right) \mathrm{d}^{3} \mathbf{v}=\int((\mathbf{v} \cdot \nabla)(\nabla \cdot \boldsymbol{U})) \frac{\delta l}{\delta \boldsymbol{\alpha}} \mathrm{d}^{3} \mathbf{v}+(\nabla \cdot \boldsymbol{U}) \int\left(\mathbf{v} \cdot \partial_{\mathbf{x}}\right) \frac{\delta l}{\delta \boldsymbol{\alpha}} \mathrm{d}^{3} \mathbf{v},
\end{aligned}
$$

and

$$
\begin{aligned}
& \int\left(\mathbf{v} \cdot \partial_{\mathbf{x}}\right)\left(\left(\partial_{\mathbf{v}} \cdot((\mathbf{v} \cdot \nabla) \boldsymbol{U})\right) \frac{\delta l}{\delta \boldsymbol{\alpha}}\right) \mathrm{d}^{3} \mathbf{v} \\
= & \partial_{\mathbf{x}} \cdot \int\left(\partial_{\mathbf{v}} \cdot((\mathbf{v} \cdot \nabla) \boldsymbol{U})\right) \mathbf{v} \frac{\delta l}{\delta \boldsymbol{\alpha}} \mathrm{d}^{3} \mathbf{v} \\
= & -\nabla \cdot \int((\mathbf{v} \cdot \nabla) \boldsymbol{U}) \frac{\delta l}{\delta \boldsymbol{\alpha}} \mathrm{d}^{3} \mathbf{v}-\int\left(\mathbf{v} \cdot \partial_{\mathbf{x}}\right)\left(\left((\mathbf{v} \cdot \nabla) \boldsymbol{U} \cdot \partial_{\mathbf{v}}\right) \frac{\delta l}{\delta \boldsymbol{\alpha}}\right) \mathrm{d}^{3} \mathbf{v} \\
= & -\int((\mathbf{v} \cdot \nabla) \nabla \boldsymbol{U}) \cdot \frac{\delta l}{\delta \boldsymbol{\alpha}} \mathrm{d}^{3} \mathbf{v}-\int \operatorname{Tr}\left((\nabla \boldsymbol{U})\left(\mathbf{v} \partial_{\mathbf{x}}\right)\right) \frac{\delta l}{\delta \boldsymbol{\alpha}} \mathrm{d}^{3} \mathbf{v} \\
& -\int\left(\mathbf{v} \cdot \partial_{\mathbf{x}}\right)\left(\left((\mathbf{v} \cdot \nabla) \boldsymbol{U} \cdot \partial_{\mathbf{v}}\right) \frac{\delta l}{\delta \boldsymbol{\alpha}}\right) \mathrm{d}^{3} \mathbf{v} .
\end{aligned}
$$

Thus, in conclusion,

$$
\int\left(£_{\mathbf{X}_{U}} \frac{\delta l}{\delta \mathbf{X}}\right)_{1} \mathrm{~d}^{3} \mathbf{v}-\int\left(\mathbf{v} \cdot \partial_{\mathbf{x}}\right)\left(£_{\mathbf{X}_{U}} \frac{\delta l}{\delta \mathbf{X}}\right)_{2} \mathrm{~d}^{3} \mathbf{v}-£_{\boldsymbol{U}} \int \frac{\delta l}{\delta \mathbf{u}} \mathrm{d}^{3} \mathbf{v}+£_{U} \int\left(\mathbf{v} \cdot \partial_{\mathbf{x}}\right) \frac{\delta l}{\delta \boldsymbol{\alpha}} \mathrm{d}^{3} \mathbf{v}=0
$$

which completes the proof. 


\section{REFERENCES}

[1] E.V. Belova, R.E. Denton, and A.A. Chan, Hybrid simulations of the effects of energetic particles on low-frequency MHD waves, J. Comput. Phys., 136(2), 324-336, 1997.

[2] A.J. Brizard and T.S. Hahm, Foundations of nonlinear gyrokinetic theory, Rev. Mod. Phys., 79(2), 421-468, 2007.

[3] A.J. Brizard and E.R. Tracy, Mini-conference on Hamiltonian and Lagrangian methods in fluid and plasma physics, Phys. Plasmas, 10(5), 2163-2168, 2003.

[4] M. Bruveris, F. Gay-Balmaz, D.D. Holm, and T.S. Ratiu, The momentum map representation of images, J. Nonlin. Sci., 21(1), 115-150, 2011. Preprint at arXiv:0912.2990.

[5] J.A. Byers, B.I. Cohen, W.C. Condit, and J.D. Hanson, Hybrid simulations of quasineutral phenomena in magnetized plasma, J. Comput. Phys., 27(3), 363-396, 1978.

[6] R. Camassa, D.D. Holm, and C.D. Levermore, Long-time shallow-water equations with a varying bottom, J. Fluid Mech., 349, 173-189, 1997.

[7] H. Cendra, D.D. Holm, M.J.W. Hoyle, and J.E. Marsden, The Maxwell-Vlasov equations in Euler-Poincaré form, J. Math. Phys., 39(6), 3138-3157, 1998.

[8] C.Z. Cheng, A kinetic-magnetohydrodynamic model for low-frequency phenomena, J. Geophys. Res., 96(A12), 159-171, 1991.

[9] C.Z. Cheng and J.R. Johnson, A kinetic-fluid model, J. Geophys. Res., 104(A1), 413-427, 1999.

[10] R. Dewar, A Lagrangian theory for nonlinear wave packets in a collisionless plasma, J. Plasma Phys., 7(2), 267-284, 1972.

[11] G.Y. Fu and W. Park, Nonlinear hybrid simulation of the toroidicity-induced Alfvén eigenmode, Phys. Rev. Lett., 74(9), 1594-1596, 1995.

[12] F. Gay-Balmaz, D.D. Holm, V. Putkaradze, and T.S. Ratiu, Exact geometric theory of dendronized polymer dynamics, preprint at arXiv:1005.2701.

[13] J.D. Gibbon and D.D. Holm, The dynamics of the gradient of potential vorticity, J. Phys. A: Math. Theor., 43, 172001, 2010.

[14] R.D. Hazeltine, D.D. Holm, and P.J. Morrison, Electromagnetic solitary waves in magnetized plasmas, J. Plasma Phys., 34, 103-114, 1985.

[15] R. Hide, Potential magnetic field and potential vorticity in magnetohydrodynamics, Geophys. J. Int., F1-F3, 125, 1996.

[16] D.D. Holm, Hamiltonian structure for Alfvén wave turbulence equations, Phys. Letter A 108, 445-447, 1985.

[17] D.D. Holm, Hamiltonian dynamics and stability analysis of neutral electromagnetic fluids with induction, Phys. D, 25(1-3), 261-287, 1987.

[18] D.D. Holm, Hamiltonian dynamics of a charged fluid, including electro- and magnetohydrodynamics, Phys. Lett. A, 114(3), 137-141, 1986.

[19] D.D. Holm and B.A. Kupershmidt, Noncanonical Hamiltonian formulation of ideal magnetohydrodynamics, Phys. D, 7(1-3), 330-333, 1983.

[20] D.D. Holm, B.A. Kupershmidt, and C.D. Levermore, Canonical maps between Poisson brackets in Eulerian and Lagrangian descriptions of continuum mechanics, Phys. Lett. A., 98, 389395, 1983.

[21] D.D. Holm, J.E. Marsden, and T.S. Ratiu, The Euler-Poincaré equations and semidirect products with applications to continuum theories, Adv. Math., 137, 1-81, 1998.

[22] D.D. Holm, J.E. Marsden, T.S. Ratiu, and A. Weinstein, Nonlinear stability of fluid and plasma equilibria, Physics Reports, 123, 1-116, 1985.

[23] C.C. Kim, C.R. Sovinec, and S.E. Parker, Hybrid kinetic-MHD simulations in general geometry, Comput. Phys. Commun., 164, 448-455, 2004.

[24] P.S. Krishnaprasad and J.E. Marsden, Hamiltonian structure and stability for rigid bodies with flexible attachments, Arch. Rational Mech. Anal., 98, 71-93, 1987.

[25] R.L. Liboff, Kinetic Theory, Springer-Verlag, 1998.

[26] R.G. Littlejohn, Variational principles of guiding centre motion, J. Plasma Phys., 29(1), 111125,1983

[27] F.E. Low, A Lagrangian formulation of the Boltzmann-Vlasov equation for plasmas, Proc. R. Soc. London, Ser. A, 248, 282-287, 1958.

[28] J.E. Marsden, G. Misiołek, J.P. Ortega, M. Perlmutter, and T.S. Ratiu, Hamiltonian Reduction by Stages, Lecture Notes in Math. 1913, Springer, 2007.

[29] J.E. Marsden, A. Weinstein, T. Ratiu, R. Schmid, and R.G. Spencer, Hamiltonian systems with symmetry, coadjoint orbits and plasma physics, Atti Accad. Sci. Torino Cl. Sci. Fis. Mat. Natur., 117(1), 289-340, 1983.

[30] P.J. Morrison, Hamiltonian and action principle formulations of plasma physics, Phys. Plasmas, 12, 058102, 2005. 
[31] P.J. Morrison, Hamiltonian field description of two-dimensional vortex fluids and guiding center plasmas, Princeton Plasma Physics Laboratory Report, PPPL-1783, 1981.

[32] P.J. Morrison, On Hamiltonian and action principle formulations of plasma dynamics, AIP Conf. Proc., 1188, 329-344, 2009.

[33] P.J. Morrison, Poisson brackets for fluids and plasmas, AIP Conf. Proc., 88, 13-46, 1982.

[34] P.J. Morrison and J.M. Greene, Noncanonical Hamiltonian density formulation of hydrodynamics and ideal magnetohydrodynamics, Phys. Rev. Lett., 45, 790-794, 1980.

[35] W. Park, E.V. Belova, G.Y. Fu, X.Z. Tang, H.R. Strauss, and L.E. Sugiyama, Plasma simulation studies using multilevel physics models, Phys. Plasmas, 6(6), 1796-1803, 1999.

[36] W. Park, S. Parker, H. Biglari, M. Chance, L. Chen, C.Z. Cheng, T.S. Hahm, W.W. Lee, R. Kulsrud, D. Monticello, L. Sugiyama, and R. White, Three-dimensional hybrid gyrokineticmagnetohydrodynamics simulation, Phys. Fluids B, 4(7), 2033-2037, 1992.

[37] D. Pfirsch, New variational formulation of Maxwell-Vlasov and guiding center theories local charge and energy conservation laws, Z. Naturforsch. A, 39a, 1-8, 1984.

[38] D. Pfirsch and P.J. Morrison, Local conservation laws for the Maxwell-Vlasov and collisionless kinetic guiding-center theories, Phys. Rev. A, 3(2), 1714-1721, 1985.

[39] D. Pfirsch and P.J. Morrison, The energy-momentum tensor for the linearized Maxwell-Vlasov and kinetic guiding center theories, Phys. Fluids B, 3(2), 271-283, 1991.

[40] P.L. Similon, A.N. Kaufman, and D.D. Holm, Oscillation center theory and pondermotive stabilization of the low-frequency plasma modes, Phys. Fluids, 29, 1908-1922, 1986.

[41] R.G. Spencer, The Hamiltonian structure of multi-species fluid electrodynamics, AIP Conf. Proc., 88, 121-126, 1982.

[42] R.G. Spencer and A.N. Kaufman, Hamiltonian structure of two-fluid plasma dynamics, Phys. Rev. A, (3)25, 4, 2437-2439, 1982.

[43] R. Takahashi, D.P. Brennan, and C.C. Kim, Kinetic effects of energetic particles on resistive MHD stability, Phys. Rev. Lett., 102, 135001, 2009.

[44] C. Tronci, Hamiltonian approach to hybrid plasma models, J. Phys. A: Math. Theor., 43, 375501, 2010.

[45] A. Weinstein, Gauge groups and Poisson brackets for interacting particles and fields, AIP Conf. Proc., 88, 1-11, 1982.

[46] D. Winske, L. Yin, N. Omidi, H. Karimbadi, and K. Quest, Hybrid simulation codes: Past, present and future - a tutorial, Lect. Notes Phys., 615, 136-165, 2003.

[47] H. Ye and P.J. Morrison, Action principles for the Vlasov equation, Phys. Fluids B, 4(4), 771-777, 1992. 